\title{
Multisensor Acoustic Tracking of Fish and Seabird Behavior Around Tidal Turbine Structures in Scotland
}

\author{
Benjamin J. Williamson $^{(\mathbb{1})}$, Shaun Fraser, Philippe Blondel, Paul S. Bell, James J. Waggitt, and Beth E. Scott
}

\begin{abstract}
Despite rapid development of marine renewable energy, relatively little is known of the immediate and future impacts on the surrounding ecosystems. Quantifying the behavior and distribution of animals around marine renewable energy devices is crucial for understanding, predicting, and potentially mitigating any threats posed by these installations. The Flow and Benthic Ecology 4D (FLOWBEC) autonomous seabed platform integrated an Imagenex multibeam echosounder and a Simrad EK60 multifrequency echosounder to monitor marine life in a $120^{\circ}$ sector over ranges up to $50 \mathrm{~m}$, seven to eight times per second. Established target detection algorithms fail within MRE sites, due to high levels of backscatter generated by the turbulent physical dynamics, limiting and biasing analysis to only periods of low current speed. This study presents novel algorithms to extract diving seabirds, fish, and fish schools from the intense backscatter caused by turbulent dynamics in flows of $4 \mathrm{~m} \mathrm{~s}^{-1}$. Filtering, detection, and tracking using a modified nearest neighbor algorithm provide robust tracking of animal behavior using the multibeam echosounder. Independent multifrequency target detection is demonstrated using the EK60 with optimally calculated thresholds, scale-sensitive filters, morphological exclusion, and frequency-response characteristics. This provides sensitive and reliable detection throughout the entire water column and at all flow speeds. Dive profiles, depth preferences, predator-prey interactions, and fish schooling behavior can be analyzed, in conjunction with the hydrodynamic impacts of marine renewable energy devices. Coregistration of targets between the acoustic instruments increases the information available, providing quantitative measures including frequency response from the EK60, and target morphology and behavioral interactions from the multibeam echosounder. The analyses draw on deployments at
\end{abstract}

Manuscript received April 13, 2016; revised October 18, 2016; accepted December 5, 2016. Date of publication January 17, 2017; date of current version October 11, 2017. This work was supported by the Natural Environment Research Council (NERC) and the Department for Environment, Food \& Rural Affairs (Defra) under Grants NE/J004308/1, NE/J004200/1, and NE/J004332/1. The work of B. Williamson was also supported by a NERC MREKEP Internship, and is now supported by an Innovate U.K. Knowledge Transfer Partnership with MeyGen Ltd. (KTP009812).

Associate Editor: D. Simons.

B. J. Williamson and B. E. Scott are with the Institute of Biological and Environmental Sciences, University of Aberdeen, Aberdeen AB24 2TZ, U.K. (e-mail: b.williamson@abdn.ac.uk; b.e.scott@abdn.ac.uk).

S. Fraser is with the School of Engineering, University of Aberdeen, Aberdeen AB24 3UE, U.K. (e-mail: s.fraser@abdn.ac.uk).

P. Blondel is with the Department of Physics, University of Bath, Bath BA2 7AY, U.K. (e-mail: P.Blondel@ bath.ac.uk).

P. S. Bell is with the National Oceanography Centre, Liverpool L3 5DA, U.K. (e-mail: psb@noc.ac.uk).

J. J. Waggitt is with the School of Ocean Sciences, Bangor University, Bangor LL59 5AB, U.K. and also with the Institute of Biological and Environmental Sciences, University of Aberdeen, Aberdeen AB24 2TZ, U.K. (e-mail: j.waggitt@bangor.ac.uk).

Digital Object Identifier 10.1109/JOE.2016.2637179 a tidal energy site in Scotland to compare the presence and absence of renewable energy structures across a range of physical and trophic levels over complete spring-neap tidal cycles. These results can be used to inform how animals forage in these sites and whether individuals face collision risks. This quantitative information can de-risk the licensing process and, with a greater mechanistic understanding at demonstration scales, its predictive power could reduce the monitoring required at future arrays.

Index Terms-Coregistration, environmental monitoring, marine renewable energy, multibeam sonar, multifrequency echosounder, target tracking.

\section{INTRODUCTION}

\section{A. Environmental Impacts of Marine Renewable}

Energy Devices

T HE drive toward sustainable energy has seen rapid development of wave and tidal stream energy, with 256 wave and 120 tidal device types [1] at either prototype or operational stage. However, relatively little is still known of any environmental and ecological effects [2], [3], although single devices are currently being scaled up to arrays and new sites are considered. Tidal stream technologies in particular add the risk of collision between animals and rotating turbine blades. This potentially represents a new mortality factor, which could significantly affect the population dynamics of many mobile marine species if significant proportions of the population are found to collide with devices [4].

Both tidal stream and wave technologies also have a more indirect effect, linked with changes to foraging efficiencies of highly mobile animals due to alterations in both near-field and far-field hydrodynamics [5]. In areas of high tidal flow, kolks (vortices within the water column) burst at the surface as "boils," which are short-lived (minutes) and of sizes comparable to the water depth [6]. These naturally forming hydrodynamic structures, with areas of horizontal and/or vertical shear, and high flow speeds of up to $4 \mathrm{~m} \mathrm{~s}^{-1}$ have the potential to aggregate, disaggregate, and disorient prey, or to provide a physical barrier within the water column, which can aid predator capture of prey [7]. The introduction of marine renewable energy devices (MREDs) has the potential for further hydrodynamic modification of flow and turbulence [8], with further possibility of prey aggregation around the structure [9]. As foraging efficiency (the capture of prey by a predator) controls both adult and juvenile survival and condition, changes in foraging efficiencies could have widespread impacts on populations [10]. 
Assessing potential population-level impacts of collisions and changes in foraging efficiency requires knowledge of animal behavior near devices and in their preferred foraging habitats; knowledge which is largely absent from the scientific literature [11]. For example, the vertical habitat preference and movements of both mobile prey (such as pelagic fish) and predators (such as diving seabirds and mammals), crucial to understanding collision risks and foraging efficiency, is generally not quantified with a high degree of accuracy during foraging events [12]. Data need to be captured at a high temporal resolution (several measurements per second, e.g., to record prey pursuit and capture) and at a high spatial resolution (centimeters, commensurate with target animal sizes) [3], [13], [14]. As regulators need to know with a high degree of certainty whether tidal and wave devices will affect populations of marine species [15], an approach that can identify and quantify changes in species-specific behavior around renewable developments is urgently required.

\section{B. Platform and Sensor Selection}

It has been recognized that novel assemblages of hydroacoustic technology on seabed-mounted platforms offer the best means to quantify concurrent animal behavior, predator-prey interactions and the hydrodynamic environment around MREDs by overcoming issues associated with alternative methods, such as vessel-based monitoring or biologging [16], [17]. The FLOWBEC platform integrated a number of instruments into a seabed frame to record information at a range of physical and trophic levels [17]. An Imagenex 837B Delta T multibeam echosounder (MBES) $\left(260 \mathrm{kHz}, 120^{\circ} \times 20^{\circ}\right.$ swath aligned with the tidal flow) was used, operating at 7-8 pings/s and tilted upward for target tracking, identification and behavioral analysis. The MBES measures the backscattering strengths (in decibels) of all targets, relative to a source level of $190 \mathrm{~dB}$ re $1 \mu \mathrm{Pa} @ 1 \mathrm{~m}$ (Patterson, personal communication, 2012). Pulse lengths vary with the range setting (e.g., $300 \mu$ s at 50-m range). The MBES was not calibrated for this study; consequently, all backscatter measurements are considered relative, not absolute.

This MBES was synchronized [17] with a Simrad EK60 multifrequency scientific echosounder $\left(38,120\right.$ and $200 \mathrm{kHz}, 7^{\circ}$ conical beamwidth), imaging vertically above the platform and used for target identification and classification, frequency response to guide species identification of fish, abundance estimates, measures of plankton, and the morphology of turbulence. The centers of each EK60 transducer were no more than $500 \mathrm{~mm}$ apart to maximize spatial comparability of the data. Each transducer used a 1024- $\mu$ s-long outgoing pulse with a ping repetition rate of $1 \mathrm{~Hz}$. The EK60 was calibrated using a 38.1-mm tungsten carbide sphere following standard procedures [18].

A SonTek/YSI ADVOcean acoustic Doppler velocimeter (ADV) measured current and turbulence at a rate of 16-20 measurements per second. A WET Labs ECO FLNTUSB fluorometer measured fluorescence (a proxy for the biomass of planktonic species) and turbidity with a measurement every 10 min. The FLOWBEC platform is self-contained with no cables or anchors, facilitating rapid deployment and recovery in high-energy sites and allowing baseline data to be gathered.
Onboard batteries and data storage enable continuous recording of a 14-day spring-neap tidal cycle. Measurements from the subsea platform are complemented with a 3-D hydrodynamic model [19], concurrent shore-based marine X-band radar [20], and ground truth wildlife observations. Full details of the FLOWBEC platform and examples from several deployments are available in [17].

Combining information from multiple instruments is highly beneficial, as it can increase coverage and sensitivity and allow one instrument to trigger the recording of another [21]. In the case of FLOWBEC, coregistration of targets seen across acoustic instruments greatly increases the information available. The instruments are positioned and oriented such that the $7^{\circ}$ conical beamwidth of the EK60 is within the $120^{\circ}$ swath of the MBES. The EK60 alone can provide quantitative metrics and patterns of target distribution [22], while coregistration of the same target on the MBES allows monitoring of concurrent behavior and predator-prey or target-MRED interactions. Targets coregistered on both instruments can be used as a training data set to aid classification of similar targets detected on a single instrument. Targets detected on a single instrument may be caused by the target either moving out of the field of view (for the EK60), being occluded by structures on the seabed (for the MBES) or by other targets, or not acquired over a few frames, for whatever reason. The co-location of both instruments on the same platform, sharing fields of view in a 2-D plane across the tidal flow, makes for more accurate target-tracking than other configurations, as demonstrated by Dell et al. in a variety of other applications [16].

\section{Processing Active Acoustic Data in High-Energy Sites}

Single beam and split beam echosounders, MBESs, and acoustic cameras have been evaluated previously for use at tidal sites [14], [22]-[24]. However, turbulence can both mask ecological targets and cause false classification. It is therefore important to identify turbulence and remove it from ecological data sets, while parameterizing ecologically relevant turbulence as a covariate and potentially a predictor of ecological activity [25].

This study demonstrates environmental monitoring of the water column in the high-energy sites around MREDs (Section II-A). Novel processing techniques have been developed to delineate and parameterize surface-connected turbulence, and extract biological targets for parameterization and tracking (Sections II-B and II-C). Coregistration of targets from an MBES and multifrequency echosounder (Section II-D) provides information gains, including robust target tracking and behavioral observations (e.g., capturing predator-prey interactions leading to the ability to quantify foraging efficiencies) with concurrent quantitative measurements of target size, distribution, and morphology. These data sets provide the quantitative data needed to investigate how fish, seabirds, and marine mammals forage within dynamic marine habitats and whether individuals face collision risks with tidal stream turbines, by providing empirical measurements of animal behavior to provide accurate estimates of parameter values in collision risk modeling. 


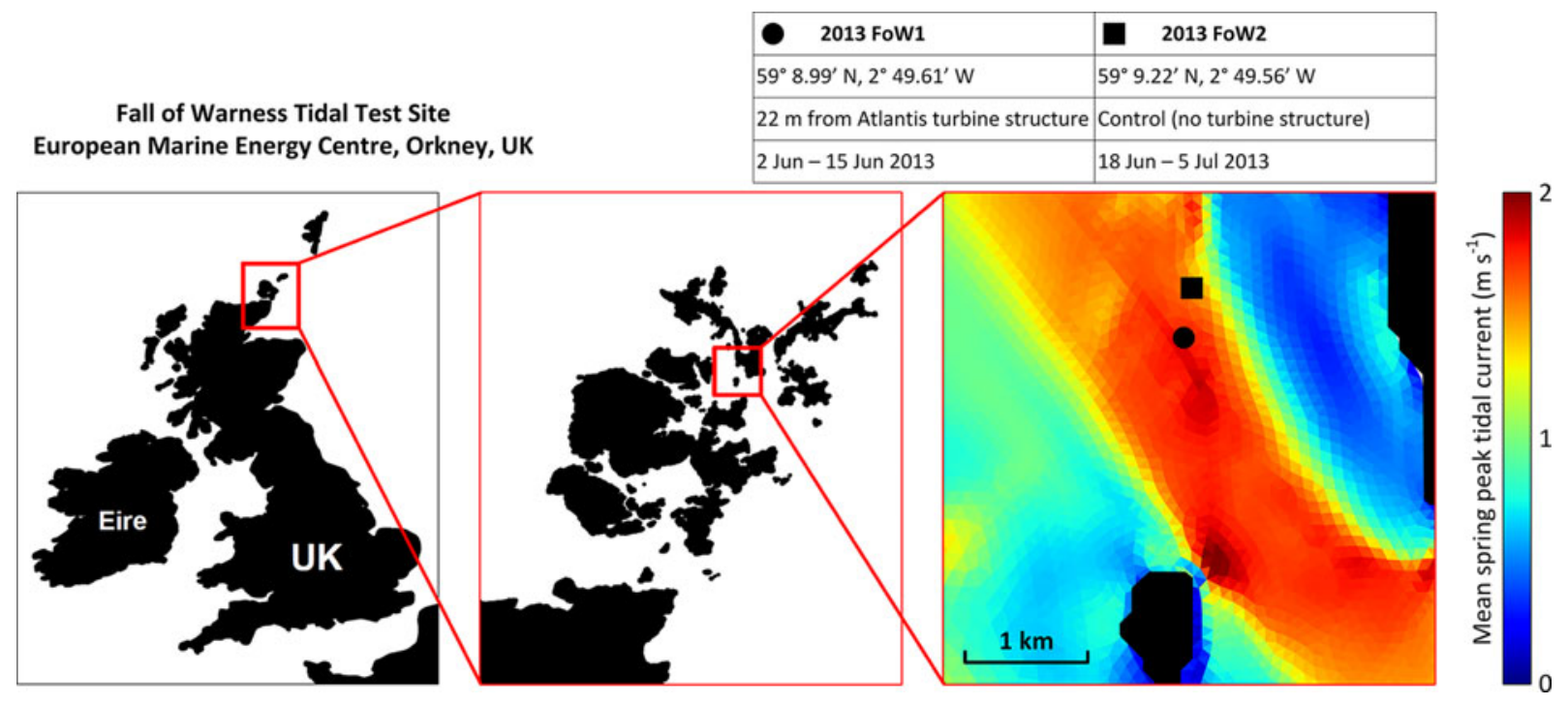

Fig. 1. Two 14-day deployments of the FLOWBEC frame (2013 FoW1 and 2013 FoW2) [17] at the EMEC Fall of Warness tidal test site are used to develop the methods to track fish and seabird behavior around tidal turbines. The map shows deployment locations in relation to mean spring peak tidal current, which is the mean of a 12-h period surrounding peak spring flow from model outputs provided by FLOWBEC project partners P. Cazenave and R. Torres, Plymouth Marine Laboratory (U.K.) [19]. Peak spring tides reach $4 \mathrm{~m} \mathrm{~s}^{-1}$.

\section{METHODS}

\section{A. Summary of Deployments}

Six deployments of the FLOWBEC frame have been completed at wave and tidal energy sites in Scotland. This paper develops the methods to track fish and seabird behavior around tidal turbines, and focuses on two deployments at the European Marine Energy Centre (EMEC) Fall of Warness (FoW) tidal site (Fig. 1). Similar methods are, of course, applicable to wave energy sites, where slower water velocities often reduce the complexity of target detection and tracking.

A deployment at a distance of $22 \mathrm{~m}$ from the center of the Atlantis AK-1000 tripod base and piling (2013 FoW1) is compared to a "control" deployment, in similar conditions, but in an area free from devices (2013 FoW2). These deployments were conducted back to back to maximize temporal comparability. Both sites had comparable depth, substrate, distance from shore, and hydrodynamic conditions to minimize natural spatial variations and to maximize spatial comparability, such that any difference observed between the two sites can be attributed to the presence/absence of the turbine structure. Both deployments targeted summer months to cover the seabird breeding season [26].

\section{B. Target Detection, Tracking, and Classification Using a Multibeam Echosounder}

Target tracking using the MBES comprises a number of steps: 1) water column delineation; 2) target detection; 3 ) target tracking; and 4) target classification (with ground truthing) and coregistration with the EK60 echosounder. These steps are presented briefly in the following sections, using the 2013 FoW1 data set as a typical example.

1) Water Column Delineation and Filtering: The first step of target detection using the MBES is delineation of the water column as a region of interest (ROI, to use the traditional

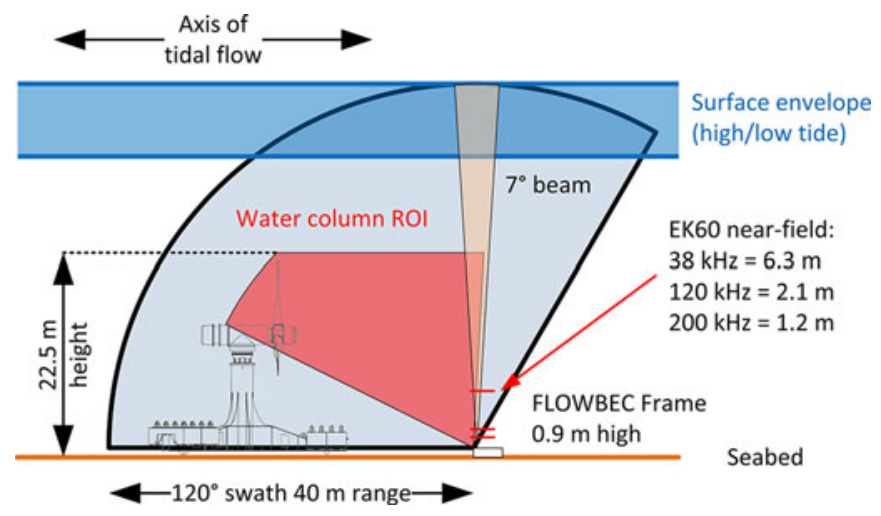

Fig. 2. The 2013 FoW1 deployment used an MBES range of $40 \mathrm{~m}$ to fully encompass the Atlantis tripod base and piling (shown to scale: the nacelle and blades were not present, but the swath included the expected full blade radius). The FLOWBEC frame was deployed approximately $15 \mathrm{~m}$ from a 4-m-high leg of the tripod base, and approximately $22 \mathrm{~m}$ from the 10-m-high center piling with the MBES swath aligned with the near-symmetric and largely bidirectional flow. The $66^{\circ} 30.4-\mathrm{m}$ range sector was cropped to a height of $22.5 \mathrm{~m}$ above the seabed to delineate targets at a vertical height overlapping the turbine structure and expected blade radius. The $7^{\circ}$ multifrequency echosounder conical beamwidth is shown for reference (orange).

notation of computer vision). The sector range is set to $30.4 \mathrm{~m}$ to exclude the reflections and the bubble layer associated with the water surface at its lowest point apparent on the MBES (spring tide, low water, bubble layer depth being also affected by winds blowing against the tide [27]). Beams in the lower $27^{\circ}$ are removed to exclude the seabed and turbine structure, and beams in the upper $27^{\circ}$ are removed to exclude reflections from the turbine structure [17]. The remaining $66^{\circ} 30.4-\mathrm{m} \mathrm{sec}-$ tor is then cropped to a height of $22.5 \mathrm{~m}$ above the seabed to study targets overlapping with the Atlantis turbine structure and expected blade radius (Fig. 2). The hazard zone of a large tidal turbine can be approximated to about two-thirds of the 


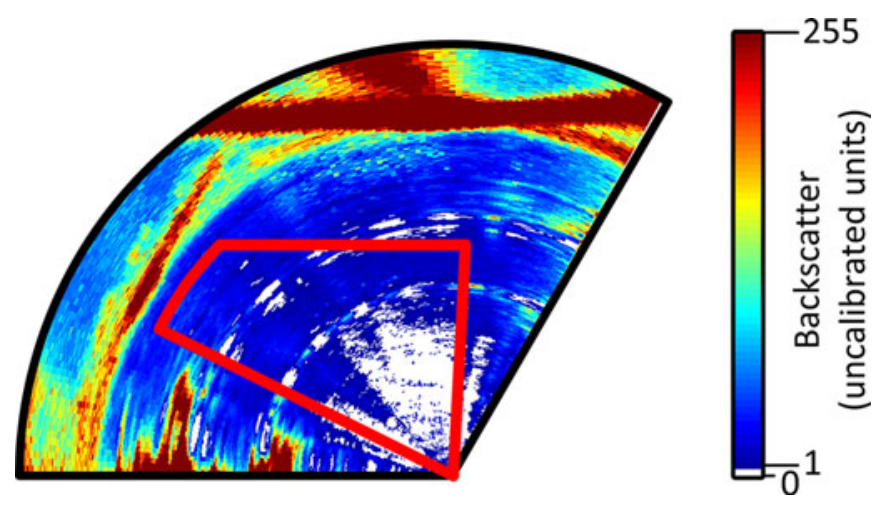

Fig. 3. A composite image, showing the maximum value observed at each ROI pixel over a 2.5 -h period reveals persistent reflections and noise. The water column ROI is shown in red. The "spoke" effect, comprising both radial and sector bands of lower intensity noise, is caused by reflections from the turbine structure and seabed. They are excluded from later analyses by setting an intensity threshold of 140 . White denotes a zero value observed throughout the entire 2.5-h period, showing masking at radial spoke intervals.

rotor-swept disc [12], and the range used here can therefore cover both the expected hazard zone and the areas beyond. The near-field minimum range of this MBES is $0.5 \mathrm{~m}$ and targets are successfully tracked at ranges $<1 \mathrm{~m}$, therefore no near-field cutoff needs to be applied.

Within the ROI, there are still several persistent reflections, caused by strong returns from the turbine structure inducing both radial and sector bands of low-amplitude noise throughout the ROI from beam sidelobes [28], [29] (Fig. 3). If not removed, these persistent reflections can be falsely detected as targets. Due to their low intensities, they can be completely removed by applying an intensity threshold of 140 to the ROI (out of a total range of $0-255$, in arbitrary units). This also removes other low-intensity spurious reflections and noise, but requires the assumption that any valid targets will have an intensity $>140$. Later plots of example targets (fish, fish schools, birds) show that this assumption is valid for the typical target strengths measured at this site. At a later stage, more sophisticated filtering (such as a more detailed mask of persistent reflections) can also be used to reduce this intensity threshold to increase the detection rates of low-intensity targets, for example, targets further than $20^{\circ}$ from the central swath axis.

Although these reflections are removed by an intensity threshold, they still cause a masking effect on targets present at the two major radial bands ("occlusion," to use the terminology of Dell et al. [16]). This is most apparent when a large target such as a school of fish passes across the MBES swath [Fig. 4(c)]. As the large continuous target passes across the radial bands, its intensity drops to near zero and its presence is masked. In effect, the ROI area is reduced by these bands of zero intensity. Target tracking is still possible, even if a target momentarily passes through these bands, using the tracking algorithms discussed in the following sections.

2) Target Detection: Algorithms were written in LabVIEW (2015, National Instruments) and MATLAB (R2013a, MathWorks) to detect targets in the ROI after applying the 140 intensity threshold with no minimum size threshold. All target detection and tracking is performed in Cartesian space, with data converted from polar (beam) coordinates to Cartesian using a nearest neighbor conversion. Targets are parameterized for computationally efficient storage and processing (a reference to the original raw MBES frame is maintained). Each target is approximated by an ellipse and stored with a number of key characteristics: its XY center, bounding ellipse area, ellipse ratio (ratio of long to short side), and ellipse orientation. The target acoustic intensity is stored as the pixel "mass" (the sum of the intensities of all pixels comprising the target), coupled with the minimum and maximum intensity of all pixels comprising the target. The number of targets per frame is also recorded.

3) Target Grouping: A dilation operation is used to ensure robust tracking of the overall movement of fish schools. Dilation adds pixels to the boundaries of objects in an image to expand shapes or pixels. This groups or aggregates closely spaced pixels by filling holes and connecting neighboring objects. As fish within a school enter and leave the side of the MBES swath (moving across swath), different individuals are detected. Additionally, as fish within the school move and change orientation, they can be momentarily detected. Given the temporal and spatial sampling resolution of the MBES, without dilation, the algorithm would incorrectly establish tracks between one individual and another within the school (or one part of a disjointed target to another due to the 140 intensity threshold), rather than the size, shape, and movement of the overall target/school.

Dilation is performed when the number of targets per frame is greater than 1, and is performed for each individual target present in the frame using a square structuring element of $8 \times$ 8 pixels, repeated 25 times. This aggregates targets within $6 \mathrm{~m}$ of each other within a frame so that multiple observations of the same target, which appear disjointed, become joined, and fish schools are parameterized as a single object. The parameters and conditions for dilation have been tuned experimentally based on manual scrutiny, to ensure dilation is only triggered when a fish school is present given the typical morphology of fish schools and individual targets observed in this survey. Although dilation is performed for target detection (to parameterize schools as a single object and to rejoin disjointed observations of the same target), the information associated with the original targets before dilation is preserved for later analysis and classificationdilation is simply used to group targets.

4) Target Tracking: Target tracking uses the parameterized list of detected targets. The aim is to identify the same individual between frames, to track its movement over time and space.

The algorithm is a modified nearest neighbor search, which seeks to establish a 1:1 relationship between all targets in the current frame to a maintained array of tracked targets [30]. The closest (nearest neighbor) target is selected as the corresponding target if its velocity is $<5 \mathrm{~m} \mathrm{~s}^{-1}$, based on the typical maximum swim speed of species present in the site [31].

A track maintenance score is used to provide tolerance of temporary nondetections of a target. Target movement and site conditions may cause a target to be momentarily not observed. Each time a target is observed, its track maintenance score is set to 15 . Targets only need to be seen once to be considered as a track candidate (no voting-in [30]). For each consecutive frame in which the tracked target is not observed, the track maintenance score is decremented (voting-out [30]). When the track maintenance score reaches zero, i.e., the tracked target 

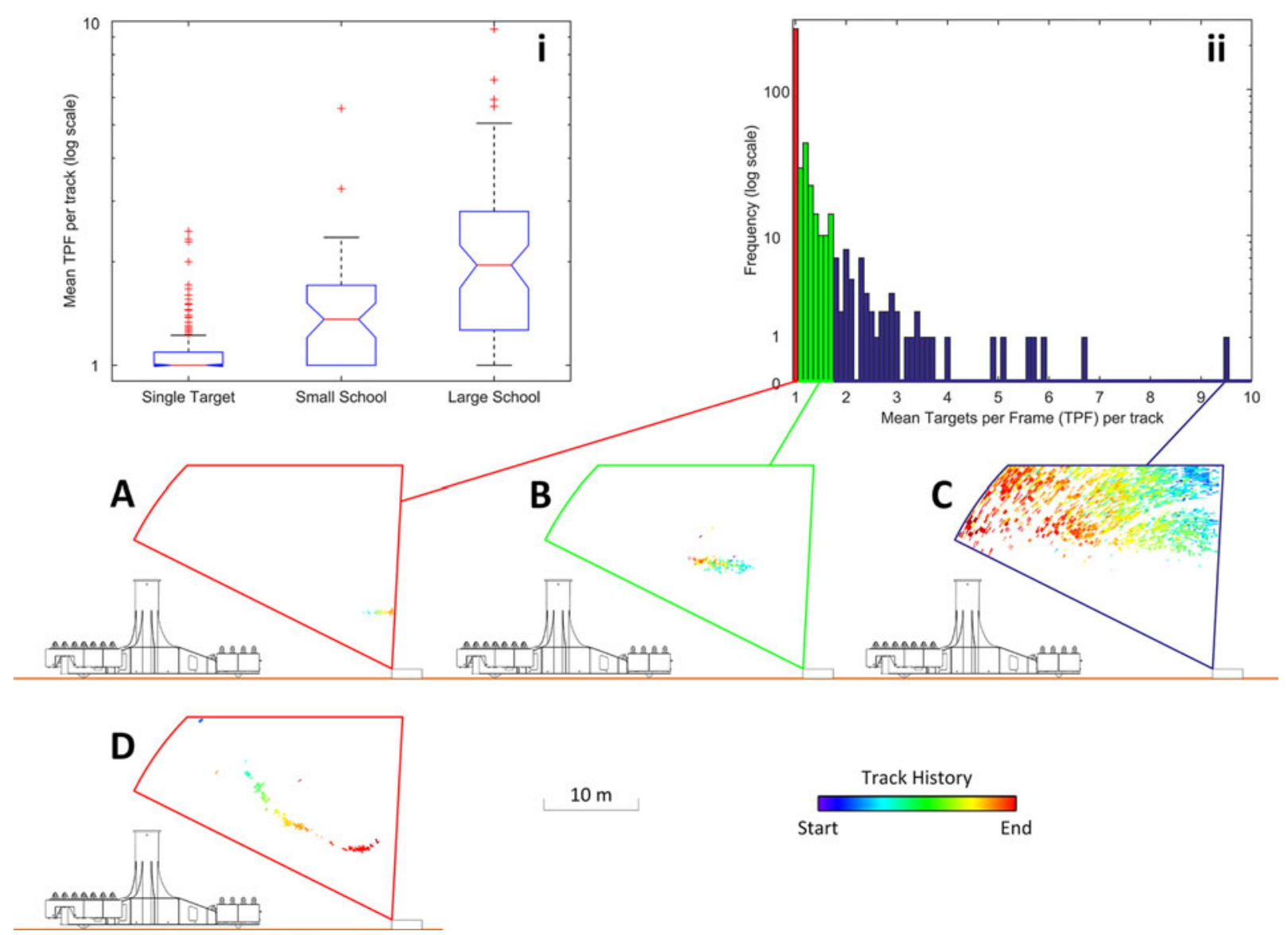

$10 \mathrm{~m}$



Fig. 4. The mean targets per frame over a track is used to classify MBES targets (i) into "single targets" [(a) and (d)], "small schools" (b), and "large schools" (c) with thresholds for each category shown by the red, green, and blue shading on the histogram (ii). Single targets are further subclassified into fish (a), diving birds (d) distinguished by their characteristic U-shaped dive profile [32] and marine mammals characterized by their large size and strong reflection. The outliers (i) reinforce the need for manual quality control of classification. The four examples target types are from the 2013 FoW1 deployment. MBES tracks are colored by time. The turbine structure is shown to scale.

has not been observed for 15 frames (approximately $2 \mathrm{~s}$ ), it is assumed that the target has left the MBES swath and the track is saved and closed. This voting-out allows targets to be momentarily not detected (for example, if they are masked by other targets, have changed attitude, or momentarily moved out of the swath). If redetected, the target is then correctly matched to the same track.

When searching for the presence of a target in a previous frame, the search area is calculated from the elapsed time between frames and the velocity threshold of $5 \mathrm{~m} \mathrm{~s}^{-1}$, i.e., search within the bounds of how far the target could conceivably have moved between frames. The search area is reduced by $5 \%$ for each historical frame using a decay function. This decay function represents the increasing uncertainty in establishing a correspondence with every frame in which the target is not observed and was tuned experimentally based on the temporal resolution of the MBES of 7 pings/s and the typical movement of targets observed in the site. If there are multiple, equally likely corresponding tracked targets, then the most recently observed tracked target is selected. If a correspondence to a tracked target cannot be established, then a new track is started using the current target observation.

The use of voting-out in combination with the decay function aims to prevent incorrect association of a new target with one seen many frames ago. All tracking values have been tuned experimentally based on manual scrutiny with the typical target detection frequency and target movement observed in the different surveys.

Various additional steps are implemented to increase tracking robustness. If there is a long duration between frames (for example, due to a synchronization error) then tracking is suspended. Additionally, when tracking targets which have been dilated (multiple targets per frame, usually fish schools), the search area for establishing correspondences between multiple frames is extended to account for the fact that the center of the fish school might not have been correctly detected (e.g., the fish school might only partially be within the MBES swath). The extension partially represents searching for correspondence from the edge of the detected school, not the acoustic centroid.

Nontracked targets are removed from later analyses in an attempt to remove spurious noise. A minimum track length of three frames is used as a threshold. The worst case scenario is detection, followed by 14 frames being voted-out while not detected, then reobservation, followed by 14 frames being votedout while not detected, before a final reobservation to form the minimum track length of three. This worst case assumes that, even at the fastest flows, targets will be present in the MBES swath for at least $4 \mathrm{~s}$. Given the $120^{\circ}$ swath aligned with the 
tidal flow, and the typical swim speeds of species present in the site [31], this assumption is deemed valid.

A minimum track length of three was selected, to allow future revisions of the tracking algorithm to use target directionality in establishing a track (i.e., do the three points trace a conceivable trajectory). Targets moving sufficiently perpendicular to the MBES swath that they are not tracked for $\geq 3$ frames are deemed rare, given the significantly bidirectional tidal flow at these sites and its alignment with the MBES swath.

The continuous track profile is stored (comprising multiple corresponded detections of an individual target or fish school) as well as average track metrics such as time and duration of occurrence, size (backscatter sum or pixel mass, area), and depth. The continuous track profile provides track speed and direction for bulk analysis, but also importantly permits examination of behavior around the turbine structure, including interactions, changes of direction (evasion), and predator-prey interactions.

Only the component of target vertical and horizontal velocity aligned with the MBES swath can be estimated; the component of across-channel/across-swath cannot be measured. However, given the often near-symmetric and largely bidirectional flow of sites selected for tidal stream turbine installations, the general preference of fauna to dive or swim with/against the flow, and the periods of greatest collision risk coinciding with the greatest along-channel water velocity, alignment of the multibeam swath with the major axis of the tidal flow maximizes the distance/time a target will be detectable within the $20^{\circ}$ across-swath beamwidth [17].

5) Target Classification: Target classification is used to guide species identification, and to allow analysis for each category of targets by time, tide, and space. Morphometric measurements (size, shape, mean backscatter and distribution of backscatter, number of targets per frame, target separation) and behavior (velocity, velocity relative to water column, directionality, vertical distribution, and intertarget interaction) are measured using the MBES, and classification performed by defining bounds for the various parameters.

At present, classification is guided by a series of key metrics and confirmed by manual quality control. This manual quality control presents the operator with the track and classification metrics in the context of the raw, unfiltered MBES data, including the surrounding time period before and after (played back faster than real time to accelerate the quality control process), and in the context of the complementary data sets (tide speed and direction, corresponding period of EK60 echogram, shore-based bird observations). Manual quality control is used not only to ensure robustness and reliability of these initial deployments of the FLOWBEC frame, but also to provide a "training" data set to confirm later development of fully autonomous classification.

The mean targets per frame over a track is used to classify MBES targets (Fig. 4), yet presence of outliers causing overlap between target categories necessitates manual quality control. These targets per frame outliers occur when a tracked target is either partially detected (e.g., part of fish school outside the ROI) or incorrectly parameterized (e.g., several distinct nonschooling targets in close proximity, or noise).
The category of single targets is further subclassified into solitary (nonschooling) fish, diving birds and marine mammals. The vertical component of target movement is used to detect the characteristic U-shaped dive of searching and foraging seabirds informed by the known diving behavior of species in the area [32], [33] and ground truthed with shore-based bird observations of diving birds. Marine mammals can be discriminated by their large size (e.g., minimum length threshold of $0.75 \mathrm{~m}$ ) and strong reflection. MBES ground-truth measurements of gray and harbor seals were conducted at a tidal site (Kyle Rhea, Scotland) using boat surveys with the FLOWBEC MBES and processing software. It is possible that seals and birds are underclassified, as the default classification of single targets is fish if the U-shaped dive or target size/backscatter does not classify a target as a bird or mammal, respectively.

\section{Target Detection and Classification Using a Multifrequency Echosounder}

Target detection and classification using the EK60 echosounder involves calibration and preprocessing, boundary delineation and data stabilization, before target detection, and parameterization. Although there are well established processing and analysis approaches for scientific echosounder data [34], the conditions recorded by the upward facing echosounders in these high-energy sites demanded a new approach to isolate ecological targets from the overwhelming backscatter generated by the turbulent physical dynamics of these extremely energetic environments. This approach is considered briefly in the following sections using the FoW data sets as an example. Full details of these methods are presented in a separate article [35].

Initial data inspection and quality control is performed with the aid of Echoview (v 5.3, Myriax Software Ltd.) but, for full flexibility, all processing and analysis was done in MATLAB using custom scripts. Initial data conversion into a MATLAB readable format used the readEKRaw MATLAB toolkit (by Rick Towler, NOAA Alaska Fisheries Science Center, Seattle, WA, USA). This approach gives full versatility in creating new tools and handling the data, although it is possible to emulate it reasonably well using Echoview alone.

In most other applications, echosounder data are gathered from a moving boat, and the boat global positioning system (GPS) track is used to convert a series of echosounder pings acquired as a function of time into a series of pings registered in space. Conversely, to characterize targets in space from a fixed seabed platform requires water velocity information. A vertical mean of water column velocity was interpolated from FVCOM data provided by FLOWBEC project partners P. Cazenave and R. Torres at the Plymouth Marine Laboratory, U.K. [19], [36]. The 15-min interval velocity data were resampled to the MBES and EK60 ping times using linear interpolation, and verified to be in phase with onboard ADV measurements of tidal velocity at $16-20 \mathrm{~Hz}$. The use of a depth-mean model speed is justified, given that sensitivity analysis identified speed as the least sensitive parameter in school detection [37].

1) Data Preparation and Water Column Delineation: Calibrated backscatter values are expressed for each of the three frequencies in the logarithmic measure of volume backscattering 
strength $\left(S_{v}\right.$ in $\mathrm{dB}$ re $\left.1 \mathrm{~m}^{-1}\right)$. The first step of data processing consists of the removal of high $S_{v}$ pixels in the near field (due to ring-down [34]) and backscatter from the sea surface.

Near-field data are excluded by a frequency-dependent constant range based on transducer characteristics [27]. This sets an effective minimum range for each frequency, approximately $6.3 \mathrm{~m}$ for $38 \mathrm{kHz}, 2.1 \mathrm{~m}$ for $120 \mathrm{kHz}$, and $1.2 \mathrm{~m}$ for $200 \mathrm{kHz}$. The $200-\mathrm{kHz}$ transducer forms the basis of many of the subsequent processing steps due to its superior effective range (smaller near field) and higher vertical resolution.

A line-picking algorithm, based on a minimum threshold for surface backscatter, is used for the exclusion of backscatter from the sea surface. This works similarly to standard seabed detection algorithms applied on downward-facing echosounder data, which rely on strong and stable $S_{v}$ at this boundary. However, the sea-surface boundary can be harder to identify accurately as the precise distinction becomes difficult during energetic periods, due to the severely disturbed nature of the sea surface and the presence of strongly reflecting, aerated water near the surface. The optimal threshold to delineate the surface effectively over the changing conditions and with minimal loss of data is selected using the method of Otsu [38], by separating the probability distribution functions of classes of pixels in an image. The resulting surface range is tested by inspection throughout the data set and by power spectrum analysis to ensure that the selected threshold yields the strongest tide-height and wave-height behavior, as expected.

The calibrated data between the near field and the detected sea surface for each frequency are checked for any issues such as interference and then are ready for further processing. These prepared data $S_{v \text { clean }}$ contain only signals resulting from ecological targets and/or from physical processes in the water column.

2) Data Stabilization and Morphological Filtering: The standard approach for identifying fish schools in EK60 data involves setting $S_{v}$ and size thresholds, applied to some combination of frequencies to define regions considered to represent schools. This is generally undertaken in established software packages using standard image processing techniques [39] and expert scrutiny [40]. These methods rely on stable acoustic background conditions for the clear differentiation of backscatter for consistent processing performance throughout the available data. However, the challenge within high current environments is that backscatter is dominated by intense physical processes varying over different length and time scales, leading to extremely unstable conditions in which to attempt reliable target identification. This means that applying sensitive thresholds to the data, which would be robust in calm conditions, leads to abundant false targets during turbulent conditions. Similarly, applying less sensitive thresholds to avoid false targets in turbulent conditions misses many targets during more stable periods. One approach would be to simply remove "bad" data, deemed too turbulent or noisy to process. However, in high-energy sites, this would involve excluding the majority of available data and biasing the final results.

An adaptive processing approach was developed to overcome this sensitivity issue, suppressing backscatter during turbulent conditions to allow effective isolation of all ecological targets using sensitive thresholds without the problem of false targets. This is conceptually equivalent to adapting thresholds to maintain the highest sensitivities possible without including false targets. The basis of this processing step is selective subtraction of the data from a scale-sensitive smoothed "background" version of the data. As the mean is calculated in the linear domain $\left(10^{S_{v} / 10}\right)$ it is highly sensitive to strong backscattering bodies such as fish schools, and so the median is used in calculations to give a stable representation of the broader conditions. For the conditions encountered at the Fall of Warness, the median is calculated as the central element in a moving window of the following dimensions: five elements in the vertical (approximately 1 -m height) and 135 elements in the horizontal (135 s). The dimensions of this window are key to the effectiveness of this step. The window should be as small as possible to have the highest resolution possible, but still more than twice as wide as the largest target of interest and high enough to give appropriate statistical stability without sacrificing depth resolution. As the largest fish schools in the data are approximately $1 \mathrm{~min}$ (60 samples) wide, and analysis of backscatter statistics demonstrates a high vertical variability in background conditions, a $5 \times 135$ window provides the optimal performance. This provides a robust smoothed approximation of the data, $S_{v} \times \times 135$ which excludes features of the scale of interest (i.e., for fish schools here, $<60 \mathrm{~s}$ ).

To selectively suppress data in response to physical conditions it is necessary to define a threshold $\lambda$ for the $S_{v 5 \times 135}$ matrix where the relatively high $S_{v}$ substantially increases the likelihood of false targets. Only when the scale-filtered data surpasses this threshold should the original data be modified to preserve functionality of sensitive target detection thresholds. If $S_{v 5 \times 135}$ is below $\lambda$, then there should be no need to suppress the data at all. The value of $\lambda$ is set at $-73 \mathrm{~dB}$ based on the scales observed for the targets of interest and the physical characteristics observed at this site. The suppression process works as follows:

$$
\begin{aligned}
& S_{v 5 \times 135} \leq \lambda \rightarrow S_{v \text { suppress }}=S_{v \text { clean }} \\
& S_{v 5 \times 135}>\lambda \rightarrow S_{v \text { suppress }}=S_{v \text { clean }}-S_{v 5 \times 135}+\lambda .
\end{aligned}
$$

This process is shown over a 12-h section of data from FoW2 which contains substantial backscatter from surface and near bed turbulent processes (Fig. 5).

This approach stabilizes the data in depth and time and gives much better performance when compared to standard $S_{v}$ and size thresholding methods for target detection, by maintaining sensitivity to strong targets in high-backscatter areas (i.e., $>\lambda$ ) without reducing sensitivity in low-backscatter areas $(<\lambda)$. However, as this approach is scale selective, any physical backscattering structure of a scale comparable to the targets of interest will be preserved, meaning an additional filter is required. In particular, wind-wave-generated clouds of air bubbles in the upper water column generate intense backscattering structures across all frequencies, extending deeper, and potentially of similar scales to fish schools. These features must be isolated and excluded from analysis or false targets will be present. 
(a) $S_{v \text { clean }}$

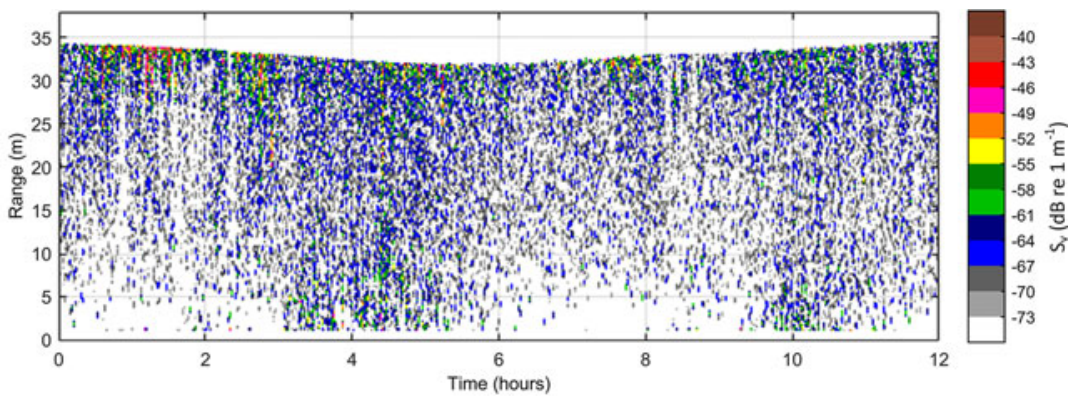

(b) $S_{V 5 \times 135}$

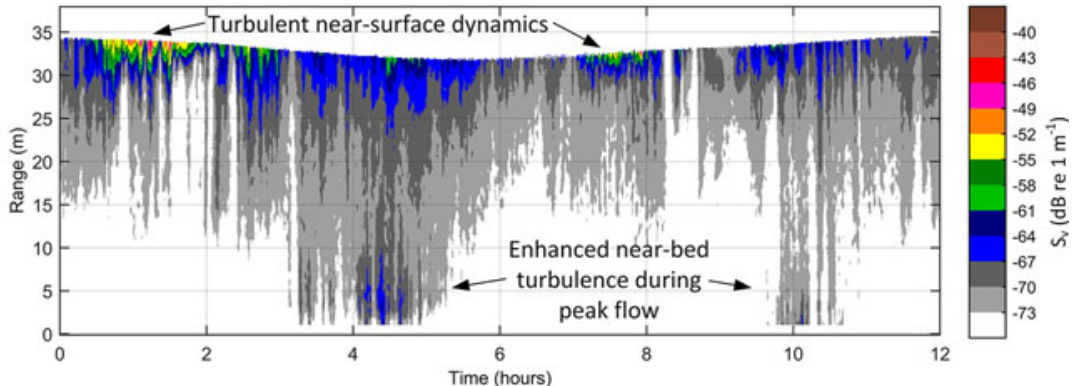

(c) $S_{V \text { suppress }}$

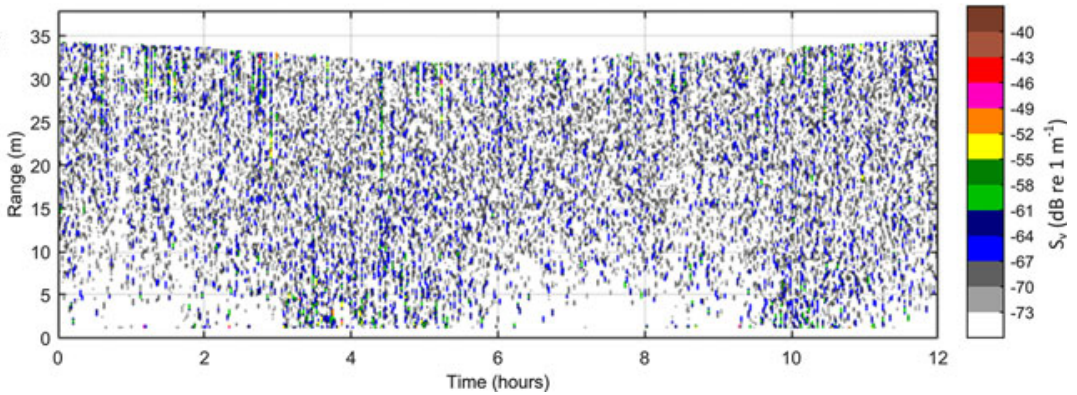

Fig. 5. Scale-dependent suppression of high backscatter regions during processing for target detection. (a) $S_{v}$ clean data from 200-kHz transducer during high turbulence in FoW2. The section begins at 12:00 on June 26, 2013. (b) Median filtered $S_{v} \times 135$ data which isolated features larger than the targets of interest corresponding to physical dynamics of the site. (c) Selectively modified $S_{v}$ suppress which stabilizes backscatter across the section, forming the basis of subsequent processing, maintaining maximum sensitivity of target detection methods without including false targets.

Such backscattering turbulent structures are morphologically isolated based on their connectivity with boundaries (e.g., the sea surface). An optimal threshold is calculated, similar to the threshold calculation for sea surface detection, which excludes these features while preserving as much data as possible. Tracing algorithms generate detailed boundary lines for these intense physical backscattering structures, and are used to remove them from further target detection steps.

Using a combined approach of adaptive scale-dependent filtering and morphological exclusion, the data can be selectively modified to give a processed, stable version $S_{v \text { stable, suitable }}$ for standard fisheries acoustics target-detection methodologies (Fig. 6). This approach maintains maximum sensitivity, despite the difficult nature of the data, by selectively modifying high backscatter regions to preserve the effectiveness of constant school detection parameters, and it is functional over even the most challenging sections of data.

3) Target Detection and Multifrequency Validation: Target detection is now performed over the processed data $S_{v}$ stable using $S_{v}$ and size thresholds. For conservative target detection, a $-55-\mathrm{dB}$ threshold on the $200-\mathrm{kHz}$ processed data set is used and a connected region with a minimum area of 10 pixels is necessary to qualify as a target (Fig. 6). In situ trawls to inform and verify these thresholds are not possible in these high-energy sites, even more so around marine energy infrastructure such as cables and structures. These thresholds were experimentally tuned, based on manual scrutiny and iterative variation to avoid false targets. Conventional vessel-based surveys typically use minimum height and length thresholds rather than the number of connected pixels; however, height and length approximations rely on the vessel having a relatively constant speed much greater than the swimming speed of the targets of interest. This assumption is not valid for echosounders mounted on stationary seabed platforms, in particular during periods of near-zero flow velocity at slack tide.

Given defined target boundaries, the final processing step involves validation of these regions using multifrequency characteristics. The mean $S_{v}$ is calculated over all the pixels in each target for each frequency to give a robust value for the mean volume backscattering strength (MVBS). The MVBS difference between frequencies is used to infer the acoustic characteristics of each target. High MVBS differences (e.g., > $10 \mathrm{~dB}$ [41]) are associated with small targets, such as plankton where size-dependent Rayleigh and resonant scattering effects are significant. In contrast, schooling fish have relatively low MVBS differences between frequencies as simple 




(b) $S_{v \text { stable }}$

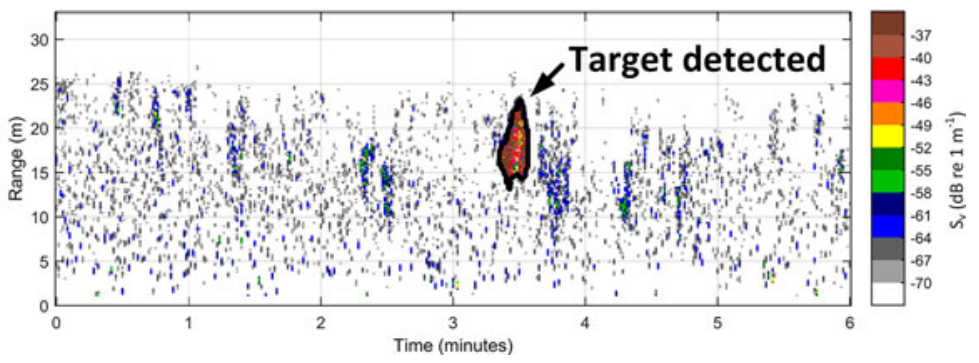

Fig. 6. A 6-min section of FoW1 data from the 200-kHz transducer showing progression from raw to fully processed stable data suitable for target detection. The section begins at 23:28:30 June 13,2013. (a) Unprocessed $S_{v}$ data showing a fish school and intense near-surface turbulence. Morphological isolation of turbulent structures is based on connectivity to the sea-surface boundary and the exclusion line is shown in black. (b) Fully processed $S_{v}$ stable data suitable for standard school detection methodologies. The perimeter of the detected target is shown in black.

geometric backscatter dominates with a relatively constant frequency response [42]. This effect is used for multifrequency validation by excluding any targets which exceed a 10-dB MVBS difference for any available frequency pair combination (e.g., $\left.\mathrm{MVBS}_{200}-\mathrm{MVBS}_{38}\right)$. Targets excluded by this process are attributed to plankton, sediment, or air bubbles. The high MVBS differences observed for excluded targets are consistent with a narrow size distribution of scattering particles. The resonant frequency of air bubbles, for example, is strongly dependent on the diameter of the bubble [27]. For the intense turbulence observed in the near surface, a broad distribution of bubble sizes is expected and suggested by the low MVBS difference values observed, which would also be consistent with fish schools for multifrequency analysis by itself. As a result, the additional processing steps are required. The majority of targets are validated in the -10 - to $+10-\mathrm{dB}$ band consistent with scattering from fish targets (Fig. 7).

4) Target Classification: Various measurements are used with a view to informing species identification and aiding target coregistration. The derivation of relevant parameters using image processing techniques is well established [40] and forms the basis of species identification [42]-[44] using discriminating characteristics. Useful parameters for classification include information on the temporal and vertical distribution of target pixels. Morphometric descriptors can then identify the shape and external structure of targets by the calculation of height, length, area, and perimeter. These can in turn be related to further descriptive characteristics such as elongation, circularity, rectangularity, and fractal dimension of the target and used to calculate an approximate target volume.

The internal structure of targets is also investigated. Target $S_{v}$ statistics such as minimum, MVBS, maximum, standard deviation, skewness, and kurtosis can give insights into the composition, structure, and behavior of targets. As already shown,

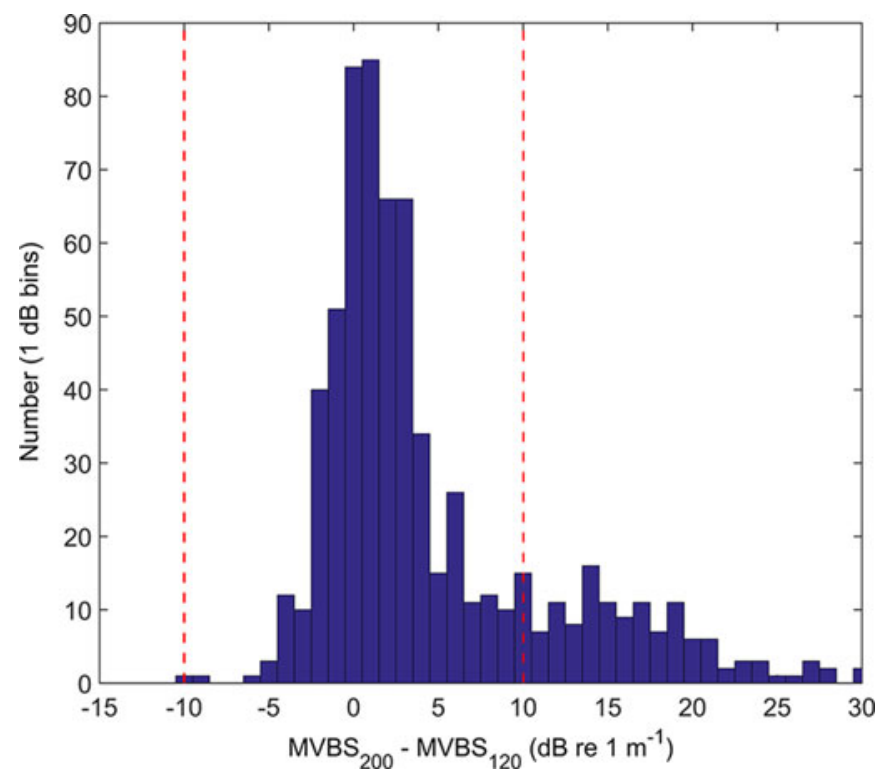

Fig. 7. Multifrequency target validation of targets from 2013 FoW1. The MVBS frequency difference for $200-\mathrm{kHz}$ data minus $120-\mathrm{kHz}$ data is shown with the number of targets (n) within 1-dB bins. Targets of interest are expected to have a relatively constant frequency response and so targets are validated when the frequency difference is within the $-10-$ to $+10-\mathrm{dB}$ band for each frequency pair combination (here shown by the red dotted lines).

the MVBS difference between frequencies is also used, and is a powerful tool in differentiating targets with different acoustic properties.

In the case of fish schools, if species composition and lengthfrequency distributions are well known, then target density and abundance can be calculated. However, the impracticalities of direct biological sampling in high-energy sites and related uncertainty in species identification mean that such outputs would be highly speculative. As a result, the descriptive 
TABLE I

ThE MBES AND EK60 HAVE COMPLEMENTARY CHARACTERISTICS AND MODES OF OPERATION, INCREASING THE DETECTION RATE. WHEN TARGETS ARE COREGISTERED BETWEEN INSTRUMENTS, THE TwO COMPLEMENTARY SETS YIELD FAR GREATER INFORMATION ON THE TARGET, GREATLY ASSISTING TARGET CLASSIFICATION



Fig. 8. Not all targets can be coregistered between instruments; the EK60 has a higher sensitivity, and the MBES has a greater detection volume as shown in the case of this figure. The diving seabird is only detectable on the second EK60 echo (after the beam has reflected off the sea surface) and would not be automatically detected by the EK60 algorithms.

parameters above are the focus of echosounder analysis and the basis for coregistration.

\section{Coregistration Between a Multibeam and Multifrequency Echosounder}

The orientation, the positioning, and the interleaving of pings from the MBES and multifrequency echosounder were chosen to insonify the same volume of water and enable detection of the same target on each instrument (Fig. 2). Coregistration is one of the greatest benefits of integrating and synchronizing the two instruments on the FLOWBEC platform. Coregistration not only adds certainty and robustness to the detection, tracking, and identification of targets, but also allows targets to be described with information from both instruments, e.g., behavior from the MBES, calibrated target strength, higher sensitivity and frequency response from the EK60 (Table I).

Coregistration is not possible for all targets; some targets might not be visible with the EK60 due to the smaller detection volume (Fig. 8), whereas lower backscatter targets might not be detectable with the MBES. Preserving targets detected on a single instrument, together with any coregistered targets, provides the most complete data set.

Coregistration aims to identify the same target on each instrument with an associated measure of certainty, by finding a single nearest neighbor match within a temporal and spatial threshold for targets observed on both instruments. This requires synchronization of both instruments, and a tolerance in time to allow for interleaved rather than simultaneous pings on each instrument. Targets may be detected on the MBES before the EK60 due to the greater swath volume in both directions, along $\left(120^{\circ}\right)$ and across swath $\left(20^{\circ}\right)$. Targets may also be seen on the EK60 before the MBES due to its higher sensitivity; for example, as a target moves and changes in orientation, it may develop a strong enough target strength that it becomes detectable on the MBES. The search bound is set to $\pm 60 \mathrm{~s}$ and it was tuned experimentally (Fig. 9), given the typical spacing in time between targets and the fact that schools are aggregated to a single object for track coregistration purposes. A spatial search bound of $5 \mathrm{~m}$ vertically is used, so that targets detected on each instrument at mean vertical separations $>5 \mathrm{~m}$ are not coregistered. This removes any outliers coexistent in time but associated with targets distinct in space. The mean target/track time from each instrument (as opposed to the time of first or last observation of the target) is used for temporal coregistration, with the possibility to use track duration and water speed as factors to vary the search thresholds.

\section{RESULTS}

\section{A. MBES Target Detection, Tracking, and Classification}

Table II lists the numbers and types of targets detected with the MBES, using the ROI and thresholds defined in Section II-B 

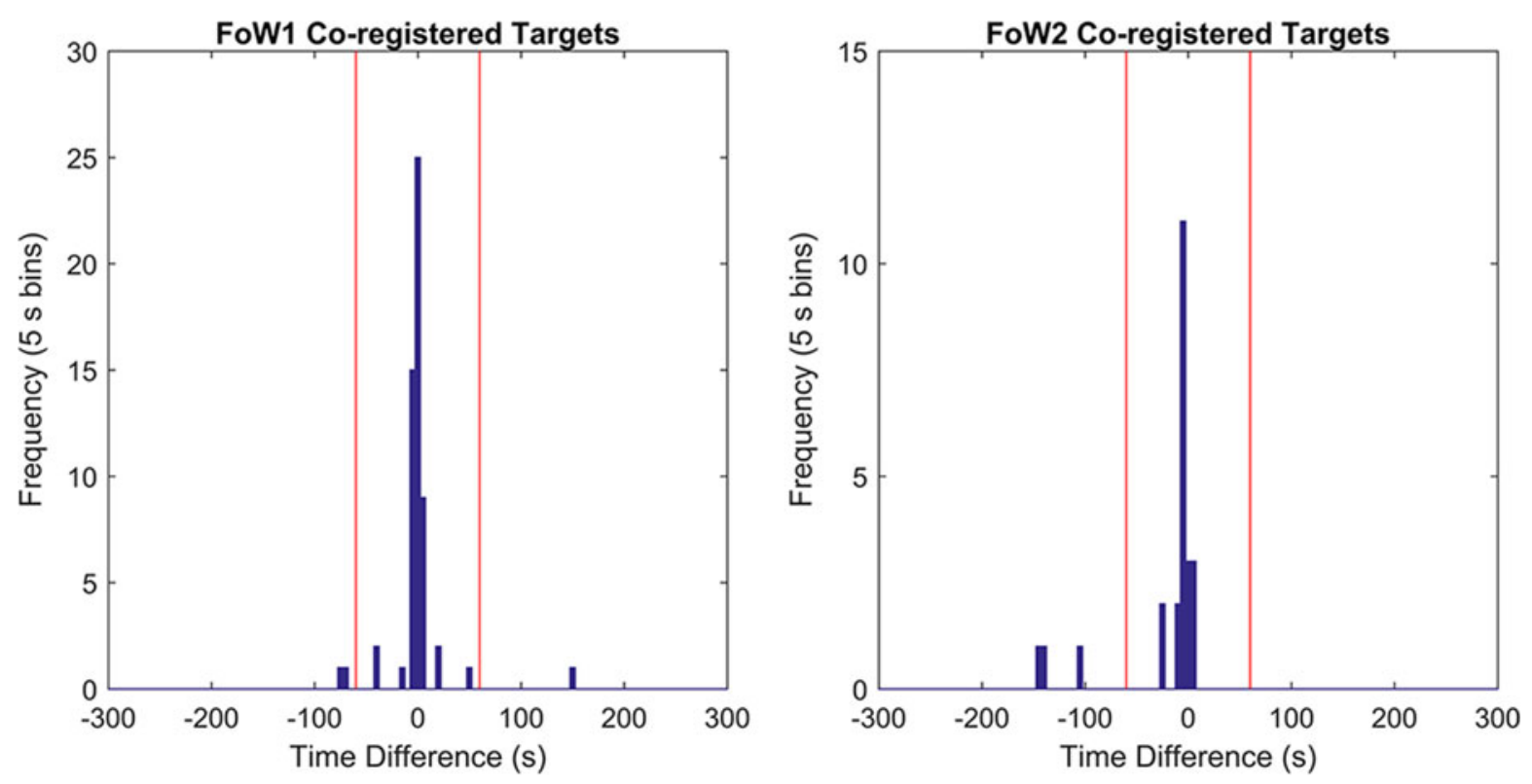

Fig. 9. The \pm 60 -s threshold (red lines) for coregistration of targets between the MBES and EK60 was tuned experimentally, based on the typical time between targets and the variation in the time of detection on each instrument. The 5-m spatial threshold between coregistered targets has already been applied in this plot. The typical spacing in time between targets removes only three targets from each deployment that are separated by $< \pm 300 \mathrm{~s}$ but $> \pm 60 \mathrm{~s}$.

TABLE II

MBES TuRbine-Height TARget Detections Using the Parameters DEFined IN THE TEXT, IN PARTiCULAR SECTION II-B

\begin{tabular}{lcc}
\hline \hline & FoW1 (264 hours) & FoW2 $(357$ hours $)$ \\
\hline Target Frames & $3657(13.85 /$ hour & $8674(24.30 /$ hour \\
& or & or \\
& $0.055 \%$ of pings $)$ & $0.096 \%$ of pings $)$ \\
Tracks - Total & $158(0.60 /$ hour $)$ & $292(0.82 /$ hour $)$ \\
Bird & $1(0.004 /$ hour $)$ & $1(0.003 /$ hour $)$ \\
Large school & $47(0.18 /$ hour $)$ & $27(0.08 /$ hour $)$ \\
Small school & $31(0.12 /$ hour $)$ & $17(0.05 /$ hour $)$ \\
Single target & $79(0.30 /$ hour $)$ & $247(0.69 /$ hour $)$ \\
\hline \hline
\end{tabular}

to cover the turbine and expected blade height $(22.5 \mathrm{~m}$ above the seabed) in both the FoW1 (Atlantis turbine base) and FoW2 (control) sites, with an intensity threshold of 140, and a minimum track length of three observations. Track durations range from 0.24 (the minimum three observations) to $78.6 \mathrm{~s}$ for large schools.

It is possible that several of the single targets are birds, but without sufficient characteristics (e.g., vertical velocity component indicating a U-shaped dive) to provide a high confidence classification based on tracked behavior alone. Although this number of confirmed turbine-height bird dives (one per site) is low, many more shallow $(<10 \mathrm{~m})$ bird dives are seen in the MBES data but not extending into the turbine height ROI. Neither of the two bird dives to the depth of the turbine height ROI coincided with ground truth observations from the shorebased observer. Instead, ground truth information for confirmed bird dives on the MBES (and EK60) was gathered for either shallower dives at the tidal sites, or during deployments of the FLOWBEC frame at the EMEC Billia Croo wave energy site.

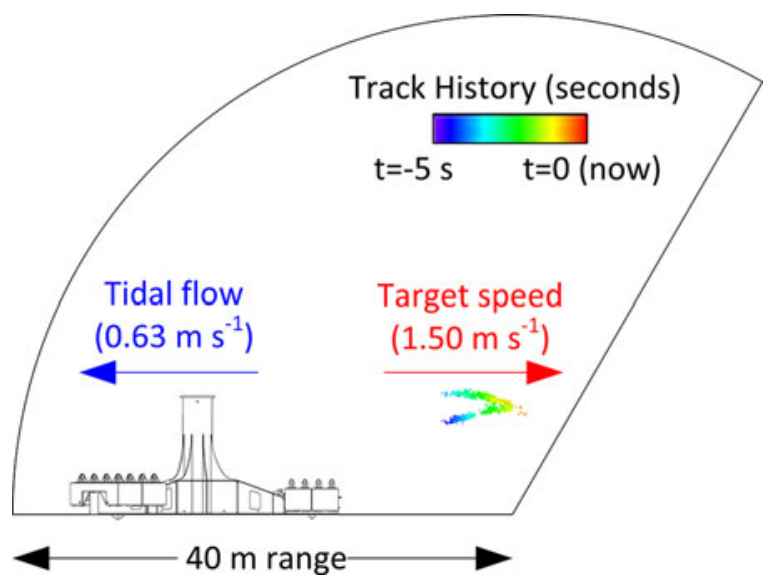

Fig. 10. This example of MBES target tracking shows the relative tracks and convergence of two fish over a 5-s period with a corresponding tidal flow of $0.63 \mathrm{~m} \mathrm{~s}^{-1}$ in the approximate direction of the turbine structure, and a target absolute speed of $1.50 \mathrm{~m} \mathrm{~s}^{-1}$ through the MBES swath, or approximately $2.1 \mathrm{~m} \mathrm{~s}^{-1}$ relative to the tidal flow. The turbine structure is shown to scale.

The fast update rate (7-8 pings/s) of the MBES allows the behavior of individual animals to be tracked. The behavior, movement, and interactions (e.g., predator-prey) of multiple targets can be visualized from inspection of tracks highlighted with multiple targets per frame (Fig. 10). The spatial coverage of the MBES also allows patterns of vertical and horizontal distribution to be investigated over time, including patterns of behavior around the MRED (Fig. 11) such as evasion.

\section{B. EK60 Target Detection}

The target detection methods described in Section II-C3 result in the following target detections (Table III) over the entire water column, and over an identical height to that 


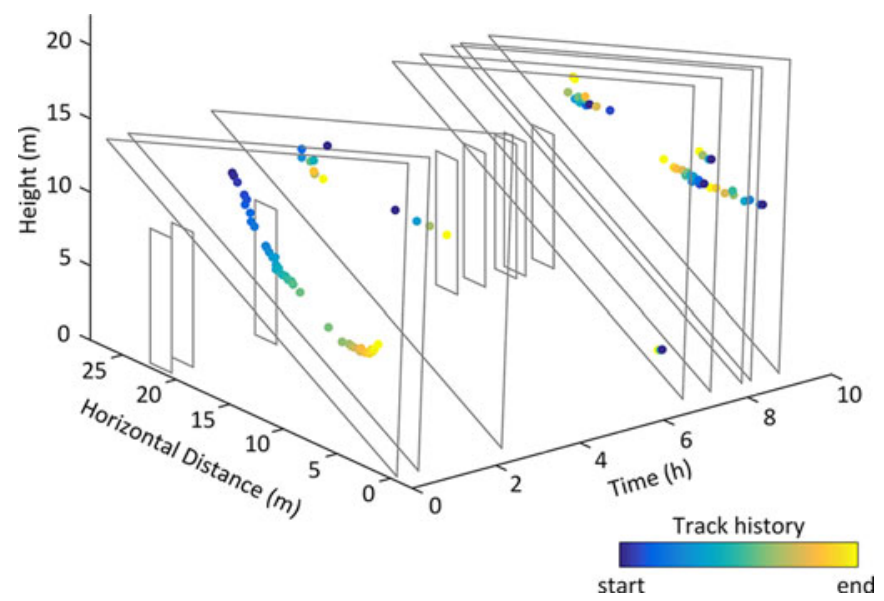

Fig. 11. An example of eight MBES tracks of a variety of birds and fish from the 2013 FoW 1 deployment, tracked in time and space over an approximately 10 -h period. Track durations in this example range from 0.25 to $14.73 \mathrm{~s}$. The first track $(t=0)$ is a diving seabird, and the remainder are either individual fish or small schools. The ROI is approximated by a triangle and the turbine central piling by a rectangle, both drawn to scale for orientation purposes.

TABLE III

EK60 TARGET DETECTIONS, USING THE PARAMETERS DESCRIBED IN SECTION II-C3

\begin{tabular}{lcc}
\hline \hline & FoW1 (273.4 hours) & FoW2 (360.7 hours) \\
\hline Full water column & $523(1.91 /$ hour $)$ & $396(1.10 /$ hour $)$ \\
Turbine height $(22.5 \mathrm{~m})$ & $355(1.30 /$ hour $)$ & $228(0.63 /$ hour $)$ \\
\hline \hline
\end{tabular}

delineated for the "turbine height" MBES ROI (22.5 m above the seabed or $21.6 \mathrm{~m}$ above the transducer). The detection of full water column EK60 targets is facilitated by robust delineation of the water column from surface noise/turbulence on a per-ping basis rather than throughout a deployment as for the MBES, and no reflections of the turbine structure and seabed on the EK60 which compromise measurements of the upper water column on the MBES. Unlike the MBES target detection results in Table II, EK60 targets cannot be robustly classified for the reasons outlined in Section II-C4. Target sizes range from single targets to schools, the largest with an observed cross-sectional area of $745.6 \mathrm{~m}^{2}$ observed over consecutive EK60 pings.

Coregistration and complementary information in Table IV details the results of temporal and spatial coregistration. MBES targets are within the turbine height ROI (Fig. 2). The 5-m target height coregistration threshold allows for the possibility of target vertical movement before/after detection on the EK60, and detection of a different acoustic center of mass between observations/instruments.

The height of detected targets on the MBES and EK60 has a strong correlation between instruments (Fig. 12, Pearson's correlation coefficient FoW $1=0.945$, FoW2 $=0.913$ ). The acoustic centroid of targets is typically observed 1-2 m lower on the MBES than on the EK60, most likely an artefact of differing beamwidth in both axes.

As an example of coregistration, a small school of fish is tracked from the 2013 FoW1 deployment next to the Atlantis turbine structure, combining behavioral information from the MBES with quantitative measures from the EK60 to increase the information available, and reduce uncertainty (Fig. 13 and Table V). Although uncalibrated, the MBES can be processed in a similar manner to the EK60 (taking a subset of the mean of the $7^{\circ}$ vertical beams), providing an additional frequency for comparison with the EK60, but with a finer temporal resolution (7 pings/s rather than $1 \mathrm{ping} / \mathrm{s}$ ) and higher spatial resolution (8 $\mathrm{cm}$ vertically rather than $19 \mathrm{~cm}$ ). This improved resolution is useful for detailed inspection of school structure. The lowintensity returns persisting over time (horizontal lines) dominating the upper part of the MBES echogram are caused by reflections from the turbine structure, also apparent on the MBES swath. The effect of ringdown on a variable per-frequency basis can be seen on the EK60. The effect of near-surface turbulence is also present and variable between the three EK60 frequencies and the MBES. The component of tidal flow oriented with the MBES swath (along swath velocity) at this time was $1.90 \mathrm{~m} \mathrm{~s}^{-1}$ in the direction from the turbine structure to the FLOWBEC frame. The target moves through the MBES swath at $0.89 \mathrm{~m} \mathrm{~s}^{-1}$, i.e., in the same direction as the water flow but slower.

\section{DISCUSSION}

This study shows that the combination of suitable acoustic instruments on a fixed seabed platform can provide information on the foraging behavior of fish, seabirds, and marine mammals at MRE sites, including predator-prey interactions and collision risks. This information can be used to investigate foraging efficiencies, and preferred foraging habitat, including preferences for depth-range and hydrodynamic conditions. Information on the temporal and vertical spatial distribution of targets can also be used to assess the collision risks with tidal stream turbines of a range of different species, by providing detailed empirical measurements of animal behavior around turbine structures for collision risk modeling.

\section{A. Target Detection and Tracking}

Established target detection approaches [39] fail within MRE sites, due to the extremely unstable acoustic conditions and the high levels of backscatter generated by the turbulent physical dynamics. The use of standard approaches would have limited analyses to the times and depths where simple target detection methods are functional. This would have rejected most of the available data, only providing a partial and biased understanding of the characteristics of targets in this environment. In this study, however, novel processing techniques have been developed to adaptively filter data, characterize turbulence, and extract biological targets for parameterization and tracking. MBES target detection and tracking has been successfully demonstrated in high-energy, acoustically noisy sites, where data are compromised by reflections and turbulence. Tracks range in size from single animals (solitary fish and diving birds) to large fish schools with an observed cross-sectional area of $745.6 \mathrm{~m}^{2}$. Using two common measures of the quality of a tracking output [16], tracks are maintained over intermittent observations due to occlusion and/or target movement with track durations up 
TABLE IV

NUMBERS OF TARGETS COREGISTERED OR INDEPENDENTLY DETECTED BY THE MBES AND EK60

\begin{tabular}{|c|c|c|c|}
\hline & $\begin{array}{l}\text { Turbine height MBES } \\
\text { tracks }\end{array}$ & $\begin{array}{c}\text { Co-registered MBES } \\
\text { and EK60 }\end{array}$ & $\begin{array}{c}\text { Turbine height EK60 } \\
\text { targets }\end{array}$ \\
\hline FoW1 & 158 & $\begin{array}{c}55 \\
(34.8 \% \text { of MBES } \\
\text { targets) } \\
\text { (15.5\% of EK60 targets) }\end{array}$ & 355 \\
\hline FoW2 & 292 & $\begin{array}{c}21 \\
\text { (7.2\% of MBES targets) } \\
\text { (9.2\% of EK60 targets) }\end{array}$ & 228 \\
\hline
\end{tabular}

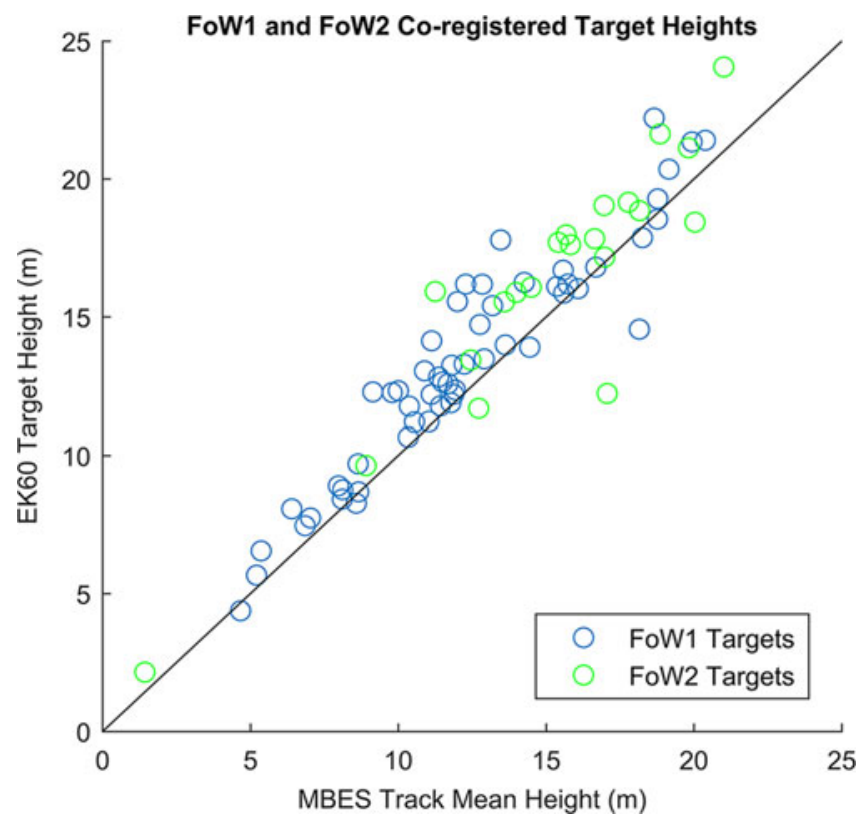

Fig. 12. Coregistration between the MBES and EK60 using temporal and spatial coregistration achieves a strong correlation of target heights measured with both instruments. A 1:1 slope line is shown for reference.

to $78.6 \mathrm{~s}$, and not only is the target center tracked, but many descriptive metrics are also tracked such as shape, perimeter, area, backscatter, distribution of backscatter, etc. This study provides a field-validated method to track multiple individuals in a complex environment, with steps toward classification, representing a cutting edge development in the application of imagebased tracking [16].

Independent multifrequency target detection has been demonstrated using a scientific echosounder and novel processing methods. Targets were isolated using optimally calculated thresholds, scale-sensitive filters, morphological exclusion, and frequency-response characteristics. The thresholds and dimensions used in these processing steps are specific to the targets of interest and physical dynamics of the site with the parameters presented here providing effective performance for isolation of fish aggregations throughout all the conditions encountered.
This process provided sensitive and reliable detection over entire deployments throughout the entire water column, preserving as much data as possible to give maximum reliable information on the full distribution and behavior of targets.

The scale requirements of the processing approach mean that this method is not suitable for continuous layers of targets, i.e., schools with a long temporal persistence at distinct depths. Similarly, detection is limited for weak targets within the most intense turbulent structures or for strong targets with no separation from the surface due to the morphological requirements in processing. Any potential targets that are not discernible from the most intense turbulent structures are excluded during processing. However, in practice, only a small proportion of data in the near-surface environment is excluded and so the potential effect on results is expected to be limited. The alternative to potential false negative detections is deemed worse, as this would falsely classify turbulent structures as near-surface fish schools, biasing results with overwhelming false positive detections of large numbers of apparent schools near the surface.

Detection efficiency is an important consideration if this methodology is to be used for environmental effect assessment, in particular, if the detection probability decreases during periods of peak flow, which could correspond with the periods of greatest collision risk. Masking of targets (false negatives) has been observed in approaches using finer-scale acoustic measurements ( $<3 \mathrm{~m}$ up/down stream) in the direct vicinity of a turbine rotor [14]. The approaches presented in this paper attempt to mitigate any effects of detection efficiency as far as possible, through the use of deployment further $(20 \mathrm{~m})$ from the turbine structure out of the immediate turbulent wake, through coregistration of multiple instruments and multiple frequencies, and through the development of novel target detection and validation algorithms. However, the potential for detection probability to decrease during periods of peak flow is inherent to the physical conditions in sites suitable for tidal stream energy extraction. Ground truthing (e.g., by trawls) is not feasible in these sites, although a dedicated experiment (e.g., investigating the detection of a calibration target of known characteristics through different hydrodynamic conditions) could be investigated. Alternative sensing modalities which could serve to provide an in situ comparison of detection functions between two different 

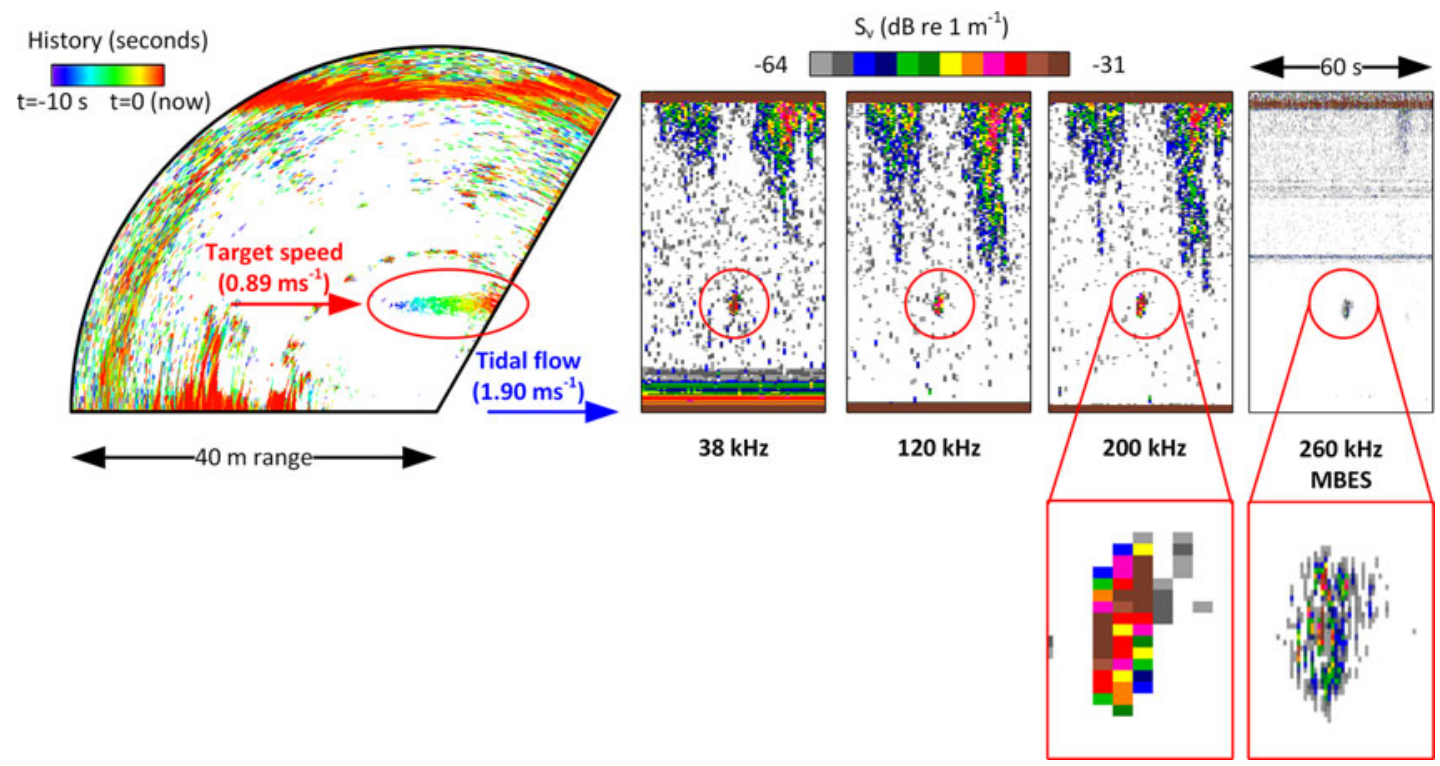

Fig. 13. Temporal and spatial coregistration is used to associate a fish school tracked on the MBES on June 12, 2013 during its departure from the Atlantis turbine structure, with the same fish school seen on the EK60. The MBES swath shows raw data from a composite image of $10 \mathrm{~s}$, color coded by time, with an intensity threshold of 40 . The EK60 raw echograms show the same period in time, without any filtering and a $S_{v}$ threshold of $-64 \mathrm{~dB}$. The rightmost plot shows the vertical $7^{\circ}$ of the MBES (the mean of seven individual beams) presented as an echogram. Although the color scale matches the EK60 echograms, the MBES units are uncalibrated.

TABLE V

Quantitative MBES AND EK60 Measures For the Fish School Shown in Fig. 13. Similar Information Is Available For All Fish Schools IDENTIFIED IN THE SURVEYS

\begin{tabular}{|c|c|c|}
\hline $\begin{array}{l}\text { Temporal } \\
\text { Co-registration: }\end{array}$ & \multicolumn{2}{|c|}{$\begin{array}{l}\text { Mean target time difference }=-1.51 \mathrm{~s} \\
\text { MBES detection prior to EK60 detection matches the observed trajectory (Fig. 13). }\end{array}$} \\
\hline Vertical Position: & MBES mean range: $11.87 \mathrm{~m}$ & EK60 mean range: $12.20 \mathrm{~m}$ \\
\hline $\begin{array}{l}\text { Spatial } \\
\text { Co-registration: }\end{array}$ & \multicolumn{2}{|c|}{ MBES mean height-EK60 mean height $=-0.33 \mathrm{~m}$} \\
\hline Target/Track: & $\begin{array}{l}\text { MBES: } \\
\text { Overall } \\
\text { Overall horizontal velocity: }-0.89 \mathrm{~m} \mathrm{~s}^{-1} \\
\text { Mean 2D angle off horizontal: } 4.50^{\circ} \\
\text { Observed target horizontal velocity in swath } \\
\text { relative to along swath tidal velocity: }-1.01 \\
\mathrm{~m} \mathrm{~s}^{-1}\end{array}$ & $\begin{array}{l}\text { EK60: } \\
\text { Height: } 2.85 \mathrm{~m} \\
\text { Length: } 6.71 \mathrm{~m} \\
\text { Observed cross-sectional area }{ }^{\mathrm{b}}: 15.33 \mathrm{~m}^{2} \\
\text { Observed perimeter: } 19.13 \mathrm{~m} \\
\text { Approximate } 3 \mathrm{D} \text { volume }: 42.93 \mathrm{~m}^{3} \\
M^{\mathrm{c}}: \mathrm{S}_{200}:-32.16 \mathrm{~dB} \text { re } 1 \mathrm{~m}^{-1} \\
M V B S_{200}-M V B S_{38}:-1.39 \mathrm{~dB} \text { re } 1 \mathrm{~m}^{-1} \\
M V B S_{200}-M V B S_{120}: 5.37 \mathrm{~dB} \text { re } 1 \mathrm{~m}^{-1}\end{array}$ \\
\hline
\end{tabular}

\title{
Contextual:
}

\author{
Water sampling: \\ 0.237 nephelometric turbidity units \\ Chlorophyll: $0.938 \mu \mathrm{g} / \mathrm{l}$ \\ Water temperature: $9.37^{\circ} \mathrm{C}$ \\ Water flow: \\ Spring (0)/neap (1) index ${ }^{\mathrm{d}}$ : 0.124 \\ Tidal flow: $2.24 \mathrm{~m} \mathrm{~s}^{-1}$, oriented N147.0 \\ Tide flow index ${ }^{\mathrm{e}}: 0.549$ \\ Along swath velocity: $-1.90 \mathrm{~m} \mathrm{~s}^{-1}$ (from \\ turbine, towards frame, flood tide) \\ Across-swath velocity: $-0.924 \mathrm{~m} \mathrm{~s}^{-1}$ (away \\ from reader in section plots above) \\ Flow offset from MBES swath: $26^{\circ} \mathrm{CCW}$
}

Tide height:

Pressure sensor: $35.35 \mathrm{~m}$

EK60 Detected surface: $35.52 \mathrm{~m}$

Tide height index ${ }^{\mathrm{f}}: 0.928$

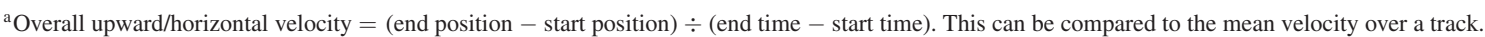

${ }^{b}$ Observed cross-sectional area is measured in the observed plane of tidal velocity combined with target movement and calculated by pixel area $\times$ number of pixels. The width of EK60 pixels for use in calculating area is calculated by the sampling interval $\times$ water velocity.

${ }^{\mathrm{c}}$ Assumes school has a cylindrical shape and that volume can be approximated by $\pi \times(\text { Height } \div 2)^{2} \times$ Length

${ }^{\mathrm{d}}$ Spring neap index is a cyclic variable between the points of highest water at each spring tide $(0 / 1)$ with 0.5 corresponding to the highest water at the intervening neap tide.

${ }^{\mathrm{e}}$ Tide flow index is a cyclic variable defined over each ebb flood cycle, based on the highest ebb direction flow (0/1) and the highest flood direction flow (0.5), with 0.25 corresponding approximately to low slack, and 0.75 approximately to high slack.

${ }^{\mathrm{f}}$ Tide height index is a cyclic variable defined over each ebb flood cycle based on the tide height measured by the frame pressure sensor. High water is defined as $0 / 1$ and low water as 0.5 . Hence 0.25 is half-way through the ebb (falling) tide height, and 0.75 is half-way through the flood (rising) tide height. A tide height and tide flow index are calculated due to the significant phase mismatch between tide height and speed at this site [7]. Rather than maximum tide speed approximately half-way between high and low water, the lowest tide height corresponds to the near maximum flow, with near-zero speeds at approximately mid-height, and still fast flowing tides at high water.
} 
sensor modes are similarly not suitable, as optical cameras are limited by turbidity and illumination [9] and tagging studies suffer from the same potential detection dependency on flow speed [45]. Instead, the current approach yields the greatest information available at this stage in these sites. Further investigation of detection efficiency is suggested as a future focus of research, and environmental effect assessment should include consideration of the potential for issues of detection efficiency.

Further processing developments informed by instrument coregistration will provide even more sensitivity and reliability, by increasing confidence in discriminating ecological targets from turbulent structures. The parameterization of ecologically relevant turbulence using appropriate metrics, measured using the coregistered echosounder and ADV, both as a time series and as turbulent features, will form the subject of future papers for analysis as covariates and potential predictors of the different trophic levels of biological activity in these sites.

\section{B. Target Coregistration}

Coregistration of targets between a multifrequency echosounder and an MBES has been demonstrated using a temporal nearest neighbor approach with a spatial threshold. After temporal coregistration with a \pm 60 -s search bound, the additional \pm 5 -m height threshold only removes additional six incorrect associations from FoW1 and ten incorrect associations from FoW2, leaving in total 55 and 21 coregistered targets at FoW1 and FoW2 respectively. Given the typical tracked target densities (an average of $0.60-1.91 / \mathrm{h}$ ), this approach was deemed robust (Fig. 9).

The thresholds and parameters for target detection, track correspondence, and coregistration have been tuned on a sitespecific basis, but can be adjusted to adapt to other sites, species, and target densities using the reasoning developed here which established each threshold. Data from further FLOWBEC deployments in other wave and tidal energy sites at EMEC and the MeyGen tidal energy site (Scotland) are being analyzed to investigate the optimization of the algorithms and thresholds for different site conditions, together with site comparisons using similar instrumentation in Admiralty Inlet, USA [22]. The coregistered monitoring techniques are now informing the environmental monitoring program for the MeyGen Tidal Energy Project in Scotland, and informing other MRE monitoring efforts worldwide.

The benefits of coregistration have been outlined (Table I), principally combining increased coverage with increased sensitivity, and combining more robust turbulence exclusion and frequency response with behavioral information and turbine-target interactions/evasion. Due to differing coverage, sensitivity, and turbulence delineation, the most complete data set results from including targets seen on a single instrument (Table IV). Coregistered targets can be used to increase certainty and validate approximate relationships between instrument metrics. These information gains can be applied in part to increase the information available from independently detected targets using the relationships between instrument metrics.
As well as the information and reliability gains from coregistration of multiple sensors, techniques such as triggering and translation are being facilitated. Triggering allows one instrument to initiate the recording of another, e.g., higher bandwidth instrument or to reduce acoustic transmissions which may modify the behavior of some animals [17], [21]. Translation allows detection and metric conversions to be established between instruments, to increase the robustness of training data sets (e.g., for classification) to then allow operation in future with a potentially reduced sensor suite, for example, lower power, lower data rates, reduced acoustic output with lower risk of disturbance, lower cost, and a physically smaller platform. For example, the possibility of future deployments without the radar, shore-based observer and a reduction in instrumentation sophistication could be investigated, using the relationships established during these deployments for detection, translation between instruments, and ground truthing.

\section{Target Classification}

Target classification from acoustic data is challenging without ground-truth information from direct sampling, such as trawls [34], which are not feasible in a tidal energy site. Instead, the classification methods in development for the FLOWBEC data sets are limited to the acoustic information available; intermittent ground-truth shore-based observations of surface targets (above water seabirds and marine mammals); and known species composition in the site [31]. Behavioral information from the MBES can be combined with the EK60 target metrics to aid classification, including comparison of dive profiles to known tag data [32], predator-prey interactions, school morphology, and relative swim speeds compared to the known swim speeds of the different species [31]. The EK60 also provides precisely defined target boundaries and calibrated multifrequency backscatter measurements to allow advanced characterization of targets to inform classification. Several target-discriminating descriptors are derived, providing information on the position, size, morphology, and internal structure of targets. Backscatter statistics and frequency response provide more information on the acoustic characteristics and distribution of scatterers within targets. Acoustic and behavioral properties of targets vary with natural conditions and the lack of direct-sampling reference data for the dynamic environments studied here makes full robust target classification particularly challenging and the subject of ongoing work.

\section{Ongoing Work}

Development of the MBES algorithms is underway to extend the ROI and reduce the target intensity threshold, coupled with more sophisticated turbulence exclusion and kinematic tracking. This can be coupled with the use of a vertical velocity profile, rather than the established standard of a depth-mean velocity [34] to enable more accurate discrimination of actively moving targets (nekton) from passively drifting particles. This will also enable more accurate length scaling of targets from acoustic measurements, and more accurate measures of target velocity relative to the water column in these high-flow shallow-water 
sites. Robustness for uncertain targets can also be added by including target metrics in the coregistration process.

\section{CONCLUSION}

The FLOWBEC project is addressing the lack of knowledge of the environmental and ecological effects of installing and operating MREDs. The FLOWBEC frame permits a continuous multiple-instrument 14-day survey from a stable platform without the cost, logistics, station keeping, and potential ecological influence of a surface platform or vessel [46], [47]. Six deployments of the FLOWBEC frame, amounting to 12 weeks in wave and tidal energy sites, have demonstrated its use as a stable and reliable instrument platform, with a deployment and recovery methodology that permits accurate and safe siting in close proximity to MRED structures. The use of a selfcontained autonomous platform allows deployments both adjacent to MREDs, and in areas free from MREDs yet subject to similar environmental conditions (bathymetry, sediment, tidal flow), to compare any ecological or environmental effects from the presence/absence of an MRED.

The present study has successfully demonstrated environmental monitoring of the water column and targets around MRED structures using novel algorithms to extract diving seabirds, fish, and fish schools from the intense backscatter caused by turbulent dynamics in flows of $4 \mathrm{~m} \mathrm{~s}^{-1}$. Active acoustic monitoring is used to track the movement and behavior of all animals within the insonified volume, irrespective of daylight, visibility, or tagged/vocalizing animals. This allows continuous surveys, 24-h a day. Filtering, detection, and tracking using a modified nearest neighbor algorithm provide robust tracking of animal behavior using the multibeam echosounder. Surveying a $120^{\circ}$ sector over ranges up to $50 \mathrm{~m}$, seven to eight times per second, individual animals and groups are tracked for durations ranging from 0.24 to $78.6 \mathrm{~s}$ over the entire water column, even in the extremely dynamic and turbulent conditions at the sites studied. Independent multifrequency target detection is demonstrated using the EK60 with optimally calculated thresholds, scale-sensitive filters, morphological exclusion, and frequencyresponse characteristics. This provides sensitive and reliable detection throughout the entire water column and at all flow speeds. Coregistration of targets between the MBES and multifrequency echosounder provides important information gains, including robust target tracking and behavioral observations (e.g., the possibility to track fine-scale behavior and predatorprey interactions) with concurrent quantitative measurements of target size, distribution, and morphology.

Using this information, the approach/departure trajectory, depth preference and interactions of birds, fish schools, and marine mammals around MRED structures can be tracked; the region investigated extends to $2 \mathrm{~m}$ from the MRED structure, and covers the water column to a height of $22.5 \mathrm{~m}$ above the seabed representing the expected blade swept area. Seabird and mammal dive profiles, predator-prey interactions, and the effect of hydrodynamic processes during foraging events throughout the water column can also be analyzed. These data sets can provide the quantitative data needed to investigate how fish, seabirds, and marine mammals forage within dynamic marine habitats, their responses to novel underwater structures, and whether individuals face collision risks with tidal stream turbines by providing empirical measurements of animal behavior to replace estimates previously used in collision risk modeling. These will form the subject of the next publications with a more biologically oriented perspective.

The combination of the sensor platform and analytical approach can support the knowledge needed by regulators for the licensing and consenting process, by providing empirical evidence of detailed animal behavior in these sites. This evidence will allow the understanding of changing foraging behavior to be used in the calculations of any changes in foraging energetics. The knowledge of the rate and depth use of the entire water column by a range of animals will assist in the calculation of the probability of collisions, as well as identify periods suitable for mitigation measures. The results can be used to guide marine spatial planning, device design, licensing, and operation, as individual devices are scaled up to arrays and new sites are considered. With a greater mechanistic understanding of how and why mobile predators use biophysical conditions at these high-energy areas for foraging, the predictive power of the outcomes may lead to a wider strategic approach to monitoring and a reduction in the level of monitoring required.

\section{ACKNOWLEDGMENT}

The authors would like to acknowledge the technical support of D. Mackay (Hydro Products Ltd., U.K.) and J. Patterson (Imagenex Technology Corp., Canada) with the MBES, E. Armstrong, V. Budreika, N. Collie, P. Copland, I. Davies, C. Hall, J. Hunter, W. Leiper, N. Morrison, B. Ritchie, C. Stewart, M. Watson, and colleagues at Marine Scotland Science, U.K., for assistance with development of the FLOWBEC platform, as well as P. Frith and P. Reddish (University of Bath, U.K.) and staff at the European Marine Energy Centre (EMEC). They also gratefully acknowledge the support of MarCRF (Marine Collaboration Research Forum). Three-dimensional hydrodynamic model data were kindly provided by FLOWBEC project partners P. Cazenave and R. Torres (Plymouth Marine Laboratory, U.K.). E. Rollings (Environment and Consents Manager, MeyGen Ltd.) is supporting the application of these techniques to monitoring the MeyGen tidal array, Scotland, as funded with an Innovate U.K. Knowledge Transfer Partnership. The constructive comments from the two anonymous reviewers and Editorial Staff are gratefully acknowledged.

\section{REFERENCES}

[1] EMEC, "List of Wave and Tidal Energy Concepts Known to EMEC," Jan. 11, 2016. [Online]. Available: http://www.emec.org.uk/marineenergy/tidal-developers/, http://www.emec.org.uk/marine-energy/wavedevelopers/.

[2] S. Benjamins et al., "Confusion reigns? A review of marine megafauna interactions with tidal-stream environments," in Oceanography and Marine Biology. vol. 53, R. N. Hughes, D. J. Hughes, I. P. Smith, and A. C. Dale, Eds., 2015, pp. 1-54.

[3] A. Copping et al., "Annex IV 2016 state of the science report: Environmental effects of marine renewable energy development around the world," 2016. [Online]. Available: http://tethys.pnnl.gov/publications/state-ofthe-science-2016. 
[4] B. Wilson et al., "Collision risks between marine renewable energy devices and mammals, fish and diving birds: Report to the Scottish executive," 2006.

[5] B. E. Scott et al., "Seabirds and marine renewables: Are we asking the right questions?," in Marine Renewable Energy Technology and Environmental Interactions, M. A. Shields and A. I. L. Payne, Eds., Amsterdam, The Netherlands: Springer-Verlag, 2014, pp. 81-92.

[6] R. N. Hughes et al., Oceanography and Marine Biology-An Annual Review, Boca Raton, FL, USA: CRC Press, 2015, vol. 53.

[7] J. J. Waggitt et al., "Quantifying pursuit diving seabirds' associations with fine-scale physical features in tidal stream environments," J. Appl. Ecol., vol. 23, pp. 1653-1666, 2016.

[8] S. Fraser et al., "Hydrodynamic and ecological impacts of turbine structures: Insights from the FLOWBEC platform," presented at the 4th Oxford Tidal Energy Workshop, Oxford, U.K., 2015.

[9] M. Broadhurst et al., "In-situ ecological interactions with a deployed tidal energy device; an observational pilot study," Ocean Coastal Manage., vol. 99, pp. 31-38, 2014.

[10] G. E. Hutchinson, An Introduction to Population Ecology. Hew Haven, CT, USA: Yale Univ. Press, 1978, Ch. 2.

[11] J. J. Waggitt and B. E. Scott, "Using a spatial overlap approach to estimate the risk of collisions between deep diving seabirds and tidal stream turbines: A review of potential methods and approaches," Mar. Policy, vol. 44, pp. 90-97, 2014.

[12] L. Hammar et al., "A probabilistic model for hydrokinetic turbine collision risks: Exploring impacts on fish," PLoS ONE, vol. 10, no. 3, 2015, DOI: 10.1371/journal.pone.0117756.

[13] B. Polagye et al., "Instrumentation for monitoring around marine renewable energy converters: Workshop final report," PNNL-23100 Pacific Northwest National Laboratory, Seattle, WA, USA, 2014.

[14] H. A. Viehman and G. B. Zydlewski, "Fish interactions with a commercialscale tidal energy device in the natural environment," Estuaries Coasts, vol. 38, pp. 241-252, 2015.

[15] M. A. Shields and A. I. L. Payne, Marine Renewable Energy Technology and Environmental Interactions, Amsterdam, The Netherlands: SpringerVerlag, 2014, doi:10.1371/journal.pone.0117756.

[16] A. I. Dell et al., "Automated image-based tracking and its application in ecology," Trends Ecol. Evol., vol. 29, pp. 417-428, 2014.

[17] B. J. Williamson et al., "A self-contained subsea platform for acoustic monitoring of the environment around marine renewable energy devicesField deployments at wave and tidal energy sites in Orkney, Scotland," IEEE J. Ocean. Eng., vol. 41, pp. 67-81, 2016.

[18] K. Foote et al., "Calibration of acoustic instruments for fish density estimation: A practical guide," ICES Cooperative Res. Rep., vol. 144, 1987.

[19] P. S. Bell et al., "Flow and benthic ecology 4D-FLOWBEC-An overview," presented at the 2nd Environmental Impacts of Marine Renewables (EIMR 2014) Conf., Stornoway, Scotland, 2014.

[20] P. S. Bell et al., "Determining currents from marine radar data in an extreme current environment at a tidal energy test site," presented at the IEEE Int. Geosci. Remote Sensing Symp., Munich, Germany, 2012.

[21] E. Cotter et al., "Challenges to Integrating Active Acoustic Sensors," presented at the 2015 Marine Energy Technol. Symp., Washington, DC, USA, 2015.

[22] L. E. Wiesebron et al., "Comparing nekton distributions at two tidal energy sites suggests potential for generic environmental monitoring," Int. J. Mar. Energy, vol. 16, pp. 235-249, 2016.

[23] G. D. Melvin and N. A. Cochrane, "Multibeam acoustic detection of fish and water column targets at high-flow sites," Estuaries Coasts, vol. 38, pp. 227-240, 2015.

[24] M. S. Bevelhimer et al., "Fish behavioral response during hydrokinetic turbine encounters based on multi-beam hydroacoustics results," presented at the 2015 Mar. Energy Technol. Symp., Washington, DC, USA, 2015.

[25] S. Fraser et al., "Turbulence, trophic interations, and sustainable energy extraction," presented at the 2nd Environ. Impacts Mar. Renewables Conf., Stornoway, Scotland, 2014.

[26] N. P. Ashmole, "Seabird ecology and the marine environment," Avian Biol., vol. 1, pp. 223-286, 1971.

[27] X. Lurton, An Introduction to Underwater Acoustics: Principles and Applications, New York, NY, USA: Springer-Praxis, 2002.

[28] C. de Moustier, "OS-CFAR detection of targets in the water column and on the seafloor with a multibeam echosounder," in Proc. OCEANS-San Diego, 2013, pp. 1-2.

[29] I. M. Parnum and A. N. Gavrilov, "High-frequency multibeam echosounder measurements of seafloor backscatter in shallow water: Part 1 Data acquisition and processing," Underwater Technol., vol. 30, pp. 3-12, 2011.
[30] S. Thrun et al., Probabilistic Robotics. Cambridge, MA, USA: MIT Press, 2005, Ch. 7.

[31] AURORA Environmental Ltd, "Environmental Statement, EMEC Tidal Test Facility Fall of Warness REP143-01-02," 2005. [Online]. Available: http://www.emec.org.uk/?wpfb_dl=54.

[32] M. Chimienti et al., "The use of an unsupervised learning approach for characterizing latent behaviors in accelerometer data," Ecol. Evol., vol. 6, pp. 727-741, 2016.

[33] R. Langton et al., "Seabird conservation and tidal stream and wave power generation: Information needs for predicting and managing potential impacts," Mar. Policy, vol. 35, pp. 623-630, 2011.

[34] E. J. Simmonds and D. N. MacLennan, Fisheries Acoustics: Theory and Practice, Hoboken, NJ, USA: Blackwell Science, 2005.

[35] S. Fraser et al., "Automatic active acoustic target detection in turbulent aquatic environments (accepted)," Limnol. Oceanogr., Methods, to be published.

[36] J. J. Waggitt et al., "Predictable hydrodynamic conditions explain temporal variations in the density of benthic foraging seabirds in a tidal stream environment," ICES J. Mar. Sci., vol. 73, no. 10, pp. 2677-2686, Jul. 1, 2016

[37] J. M. Burgos and J. K. Horne, "Sensitivity analysis and parameter selection for detecting aggregations in acoustic data," ICES J. Mar. Sci., vol. 64, pp. 160-168, 2007.

[38] N. Otsu, "A threshold selection method from gray-level histograms," IEEE Trans. Syst. Man Cybern., vol. 9, pp. 62-66, 1979.

[39] M. Barange, "Acoustic identification, classification and structure of biological patchiness on the edge of the Agulhas Bank and its relation to frontal features," South African J. Mar. Sci., vol. 14, pp. 333-347, 1994.

[40] D. Reid et al., "Standard protocols for the analysis of school based data from echo sounder surveys," Fisheries Res., vol. 47, pp. 125-136, 2000

[41] M. Kang et al., "Effective and accurate use of difference in mean volume backscattering strength to identify fish and plankton," ICES J. Mar. Sci., vol. 59, pp. 794-804, 2002.

[42] R. J. Korneliussen et al., "Acoustic species identification of schooling fish,” ICES J. Mar. Sci., vol. 66, pp. 1111-1118, 2009

[43] H. J. Lu and K. T. Lee, "Species identification of fish shoals from echograms by an echo-signal image processing system," Fisheries Res., vol. 24, pp. 99-111, 1995.

[44] J. K. Horne, "Acoustic approaches to remote species identification: A review," Fisheries Oceanogr., vol. 9, pp. 356-371, 2000.

[45] A. M. Redden et al., "Acoustic tracking of fish movements in the Minas Passage and FORCE demonstration area: Pre-turbine baseline studies (2011-2013)," Acadia Centre for Estuarine Research, Tech. Rep. No. 118, Acadia Univ., Wolfville, NS, Canada, 153 pp., 2014.

[46] M. L. Tasker et al., "Counting seabirds at sea from ships: A review of methods employed and a suggestion for a standardized approach," The Auk, vol. 101, pp. 567-577, 1984.

[47] P. Schwemmer et al., "Effects of ship traffic on seabirds in offshore waters: Implications for marine conservation and spatial planning," Ecol. Appl., vol. 21, pp. 1851-1860, 2011.

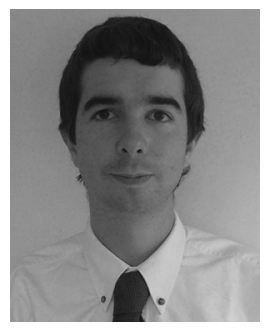

Benjamin J. Williamson received the B.Eng. degree (honors) in electrical and electronic engineering, the M.Sc. degree (with distinction) in mechatronics, and the Ph.D. degree from the University of Bath, Bath, U.K., in 2006, 2007, and 2013, respectively. His dissertation focused on novel hybrid-ROV/AUVs for habitat mapping of kelp beds using multibeam sonar and video in near-shore environments.

He is currently a Research Fellow at the University of Aberdeen, Aberdeen, U.K., working on the FLOWBEC Project, which investigates the environmental and ecological effects of wave and tidal energy devices. His research interests include autonomous underwater vehicle (AUV) development, instrument integration, and algorithm development for sonar processing and target tracking. 




Shaun Fraser received the first class B.Sc. degree (Hons.) in geophysics in 2013 from the University of Edinburgh, Edinburgh, U.K., where his dissertations were on the development of cellular automata to model upland river erosion and the study of gravity variations to investigate geological faults.

He joined Xodus Group in 2013 as an environmental consultant before beginning his current work towards the Ph.D. degree at the University of Aberdeen, Aberdeen, U.K., where he is investigating the physical and ecological dynamics of high energy marine environments. His research involves the application of marine active acoustics to analyze turbulence in coastal regions, and the development of acoustic processing methods to study biological targets for environmental monitoring around marine renewable energy infrastructure.

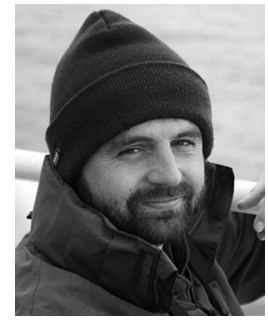

James J. Waggitt received the first class B.Sc. degree in marine biology and coastal ecology and the M.Res. degree with distinction in marine biology from the University of Plymouth, Plymouth, U.K., in 2009 and 2010, respectively, and the Ph.D. degree in marine ecology from the University of Aberdeen, Aberdeen, U.K., in 2015.

He is now a Research Officer at Bangor University, Menai Bridge, U.K. His research focuses on the behavioral and environmental factors determining the foraging distribution of seabirds and cetaceans. This research has included the combination of biologging, observational surveys, and hydrodynamic model data to test hypothesis-driven questions concerning when and where foraging seabirds and cetaceans are found.

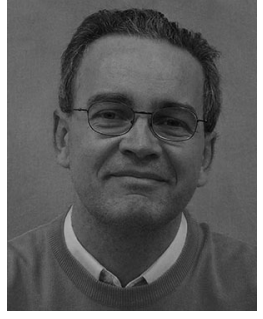

Philippe Blondel received the DEUG-A degree in physics from the University of Rouen, Mont-SaintAignan, France, in 1985, the Maitrise degree in physics from the University of Paris-XI, Orsay, France, in 1987, and the Ph.D. degree in physics and remote sensing from the University of Paris-VII, Paris, France, in 1992.

He worked at the University of Washington on sonar imaging at mid-ocean ridges as a Lavoisier Fellow, carrying on this work as a NERC Fellow at the Institute of Oceanographic Sciences, Deacon Laboratory, and the Southampton Oceanography Centre (U.K.). He moved to the University of Bath, Bath, U.K., in 1999, where he is now Senior Lecturer in Physics and Deputy Director of the Centre for Space, Atmosphere, and Ocean Science. He has written several research textbooks including the Handbook of Sidescan Sonar (New York, NY, USA: Springer-Verlag, 2009). His research interests include acoustic imaging of marine habitats, underwater noise and anthropogenic impacts, and acoustic monitoring of Arctic processes.

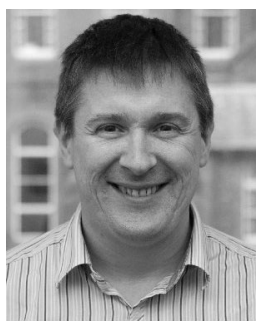

Paul S. Bell received the B.Sc. degree (honors) in physics and electronics from the School of Physics and Astronomy, University of St. Andrews, Fife, Scotland, in 1992 and the Ph.D. degree from the School of Ocean Sciences, University of Wales, Bangor, U.K., in 2005, with a dissertation on the remote determination of bathymetric changes using ground-based radar.

Since 1992, he has been with the National Oceanography Centre (previously known as the Proudman Oceanographic Laboratory), Liverpool, U.K., developing a variety of acoustic and radar techniques to study flow and sediment dynamics at coastal sites. He is currently working on the use of groundand vessel-based marine radar to map bathymetry, currents, and other hydrodynamic properties at coastal sites.



Beth E. Scott received the B.Sc. degree in marine ecology from Simon Fraser University, Burnaby, BC, Canada, in 1984, the M.Sc. degree in fisheries oceanography from the University of British Columbia, Vancouver, BC, Canada, in 1990, and the $\mathrm{Ph} . \mathrm{D}$. degree from the University of Aberdeen, $\mathrm{Ab}$ erdeen, U.K., in 2003, which focused on creating individual-based models (IBMs) for commercial fish species.

She was a Research Fellow at FRS Marine Lab. She is now a Reader at the University of Aberdeen where she conducts multidisciplinary research using expertise in marine ecology, oceanography, and fisheries. Her research identifies general rules in biophysical oceanographic processes that lead to the creation of hotspots of predator-prey activity. 